\title{
Restoring ancestral phenotypes by reduction of plasticity is a general pattern in gene expression during adaptation to different stressors in
}

\section{Tribolium castaneum}

Eva L. Koch ${ }^{1}$ and Frédéric Guillaume ${ }^{1}$

${ }^{1}$ Department of Evolutionary Biology and Environmental Studies, University of Zürich, Winterthurerstr. 190, 8057 Zürich, Switzerland

Corresponding author:

Prof. Dr. Frédéric Guillaume

tel: ++41 446356623

frederic.guillaume@ieu.uzh.ch 


\section{Abstract}

Plasticity and evolution are two processes individuals to respond to environmental change, but

3 how both are related and impact each other is still controversial. We studied plastic and evolutionary responses in gene expression of Tribolium castaneum after exposure to new environments that differed from ancestral conditions in temperature, humidity or both. Using experimental evolution with ten replicated lines per condition, we were able to demonstrate adaptation after 20 generations. We measured gene expression in each condition in adapted

8 selection lines and control lines to infer evolutionary and plastic changes. We found more 9 evidence for changes in mean expression (shift in the intercept of reaction norms) in adapted 10 lines than for changes in plasticity (shifts in slopes). Plasticity was mainly preserved and was

11 responsible for a large part of the phenotypic divergence in expression between ancestral and 12 new conditions. However, we found that genes with the largest evolutionary changes in 13 expression also evolved reduced plasticity and often showed expression levels closer to the

14 ancestral stage. Results obtained in the three different conditions were similar suggesting that

15 restoration of ancestral expression levels during adaptation is a general evolutionary pattern. We

16 increased the sample size in the most stressful condition and were then able to detect a positive

17 correlation between proportion of genes with reversion of the ancestral plastic response and 18 mean fitness per selection line.

\section{Introduction}

22 Whenever facing environmental change, populations can adapt to new phenotypic optima by

23 plasticity and evolution. Plasticity is the ability of a single genotype to produce multiple

24 phenotypes as a function of the environment. It is often seen as an immediate response of 25 individuals to changes in their environment. In contrast, evolution requires a change in allele 
26 frequencies within a population. This process occurs over several generations and represents a

27 more long-term response, which can result in local adaptation. It is still not well understood how

28 these two processes are related and interact with each other (de Jong, 2005; Forsman, 2015;

29 Ghalambor, McKay, Carroll, \& Reznick, 2007; Price, Qvarnström, \& Irwin, 2003; Wund, 2012).

31 Plasticity does not affect a population's genetic composition and should thus not have long-term

32 consequences in the following generations. However, it changes the distribution of phenotypes

33 on which selection can act (de Jong, 2005; Pfennig et al., 2010; Pigliucci, 2005; Price,

34 Qvarnstrom, \& Irwin, 2003). If plasticity perfectly matches the new phenotypic optimum, it

35 prevents evolution since no genetic changes are required and selection is prevented (Ghalambor

36 et al., 2007). On the other hand, plasticity is also crucial for a population's persistence and can

37 reduce the costs of selection (Chevin, Lande, \& Mace, 2010; Pavey, Collin, Nosil, \& Rogers, 2010).

38 It prevents extinction and protects populations from bottleneck effects, thereby maintaining a

39 higher genetic variation on which subsequently selection can act (Fitzpatrick, 2012; Pfennig et

40 al., 2010; Massimo Pigliucci, 2005). There is both, theoretical (Chevin et al., 2010; Draghi \&

41 Whitlock, 2012; Fierst, 2011) and empirical work (Schaum, Rost, Millar, \& Collins, 2013),

42 demonstrating that more plastic populations exhibit faster evolution. The benefits of plasticity

43 for persisting in new habitats were also demonstrated in invasive species (Molina-Montenegro,

44 Peñuelas, Munné-Bosch, \& Sardans, 2012; Pichancourt \& van Klinken, 2012; Yeh \& Price, 2004).

46 The extent of plasticity can be represented as a reaction norm (Scheiner, 1993), which is the

47 phenotypic trait value as a function of an environmental variable. Evolution can affect the

48 reaction norm in two ways: The intercept can be shifted corresponding to a change in the mean

49 phenotypic value. Alternatively, the slope of the reaction norm, i.e. the plasticity, can be

50 changed. Thus, plasticity itself can also be subject to evolution given that there is sufficient 
51 genetic variation in reaction norms (Garland \& Kelly, 2008; Nussey, Postma, Gienapp, \& Visser,

52 2005).

54 Dependent on whether plastic responses are adaptive and increase fitness of an individual,

55 different patterns describing the relationship between ancestral plasticity and evolution are

56 possible. If plastic responses are adaptive, but not sufficient to reach the phenotypic optimum,

57 evolution should work in the same direction as the plastic response (referred to as Baldwin effect

58 (Crispo, 2007) or cogradient variation (Conover, Duffy, \& Hice, 2009). In this case, selection may

59 favour the most plastic individuals, causing evolved populations to exhibit a higher plasticity than

60 their ancestors (Crispo 2007; Lande 2009). Another possible outcome is genetic assimilation: An

61 initially environmentally induced phenotype can get fixed by a loss of plasticity and becomes

62 continuously expressed even in the ancestral environment (Levis \& Pfennig, 2016; Pigliucci,

63 Murren, \& Schlichting, 2006). In contrast, if plastic responses are maladaptive, we expect to

64 observe evolutionary changes opposite to plasticity (countergradient variation ((Conover et al.,

65 2009) or genetic compensation (Grether 2005)). Maladaptive plasticity was proposed as a

66 possible mechanism promoting evolution since it moves phenotypes further away from the

67 optimum and thereby increases the strength of selection on the phenotype (Ghalambor et al.,

68 2007). Both co-gradient (Barton, Sunnucks, Norgate, Murray, \& Kearney, 2014; Conover et al.,

69 2009) and counter-gradient (Conover et al., 2009; Ghalambor et al., 2015; Laugen, Laurila,

70 Räsänen, \& Merilä, 2003) evolutionary changes have been found, indicating that adaptive and

71 maladaptive plasticity are common. Reversion of ancestral plasticity occurs more frequently (Ho

72 \& Zhang, 2018), indicating that plastic responses are often not beneficial for long-term

73 adaptation. 
Plastic responses in physiology, behaviour or morphological traits are often initiated by changes in gene expression (Hodgins-Davis \& Townsend, 2009; Wray, 2007). The transcriptome represents a direct link between genotype and phenotype making it particularly interesting to study the interplay between plasticity and evolution. Transcription is highly plastic and modulating expression levels is an important part of an organism's physiological adjustment to

80 environmental change (Gibson, 2008; McCairns \& Bernatchez, 2009). On the other hand, there

81 are also many studies demonstrating evolutionary divergence in gene expression between locally

82 adapted populations (Alvarez, Schrey, \& Richards, 2015; Guo et al., 2016; Romero, Ruvinsky, \&

83 Gilad, 2012; Townsend, Cavalieri, \& Hartl, 2003; Whitehead \& Crawford, 2006). Gene expression

84 may even evolve more rapidly than changes in proteins since mutations affecting the magnitude

85 of expression are less likely to be deleterious than changes in protein structures (Carroll, 2005;

86 Wray, 2007). However, it is not clear how fast plasticity in gene expression can change. Some

87 studies reported changes in plasticity in few genes after adaptation to new conditions (Morris et

88 al., 2014; Passow et al., 2017; von Heckel, Stephan, \& Hutter, 2016), whereas others found only

89 limited evolution of plasticity (Yampolsky, Glazko, \& Fry, 2012) or less than expected (Huang \&

90 Agrawal, 2016).

92 In our study, we used whole transcriptomes to understand the interplay between plasticity and

93 evolution at the gene expression level during adaptation to new environments with the model

94 organism Tribolium castaneum (red flour beetle) using an experimental evolution approach.

95 With RNA-seq, we quantified plastic and evolutionary changes in gene expression and their

96 contribution to the total phenotypic divergence between populations inhabiting different

97 environments. More specifically, we were interested to test whether the same genes exhibited

98 both evolutionary and plastic changes in a new environment and whether evolved changes were

99 in the same direction as their ancestral plasticity. In doing so, we could test how plasticity 
100 affected evolution. We tested whether plasticity prevented evolution, or whether maladaptive

101 plasticity increased the strength of selection and promoted evolution. Next, we tested how

102 evolution affected gene expression reaction norms, i.e. whether adaptation to new conditions

103 was achieved by changes in slopes or intercepts.

\section{$107 \quad$ Material and Methods}

108 Animal rearing, experimental evolution

109 We used the Tribolium castaneum Cro1 strain (Milutinović, Stolpe, Peuß, Armitage, \& Kurtz, 110 2013), collected from a wild population in 2010 and adapted to lab standard conditions $\left(32^{\circ} \mathrm{C}\right.$,

$11170 \%$ relative humidity (r.h.)) for more than 20 generations. Beetles were kept in $24 \mathrm{~h}$ darkness on 112 organic wheat flour mixed with $10 \%$ organic baker's yeast. We sterilized flour and yeast by

113 heating them for $12 \mathrm{~h}$ at $80^{\circ} \mathrm{C}$ before use. To test for adaptation to new environmental conditions

114 we used replicate lines and exposed them to three treatment and control (CT) conditions. The 115 conditions in the treatments were: Dry (D): $33^{\circ} \mathrm{C}$ and $30 \%$ r. h.; Hot (H): $37^{\circ} \mathrm{C}$ and $70 \%$ r. h.; Hot-

116 Dry (HD): $37^{\circ} \mathrm{C}$ and $30 \% \mathrm{r}$. h. To generate replicate lines, we used 120 individuals (60 females

117 and 60 males in the pupal stage) and placed them into a vial containing $80 \mathrm{~g}$ medium. We

118 produced six lines per selection regime (treatments plus control), resulting in a total of 24 lines.

119 For each new generation, we randomly collected 120 pupae and placed them into a new vial.

120 After seven to ten days, in which the pupae became adults, mated and laid eggs, adult beetles

121 were removed by sieving the medium. We waited until the next generation (eggs/larvae in the 122 medium) had reached the pupal stage and again collected 120 pupae per line to establish the 123 next generation. This is similar to natural selection since individuals, depending on their fitness, 124 do not contribute equally to the next generation. In generation 15 we produced additional mixed 
125 lines to prevent loss of genetic diversity by gene drift and inbreeding, which might impede

126 adaptation: We mixed the six replicate lines of each selection regime in equal proportions (20

127 individuals from each replicate line) four times, resulting in four mixed lines with 120 individuals

128 each. In total we had 39 lines: six normal and four mixed lines per selection regime (one line in D

129 became extinct). The transplant experiment to test for adaptation was conducted in generation

$130 \quad 22$.

132 Reciprocal transplant and fitness assay

133 Before testing for adaptation, all lines stayed for two generations in the same condition to 134 remove potential maternal or epigenetic effects (Supporting information, Figure S1): Beetles of

135 generation 20 from all selection lines were transferred to control conditions, in which they 136 stayed for one week to mate and lay eggs. After removal of the adults, we waited until their 137 offspring had reached the pupal stage and separated males and females. These individuals 138 (generation 21) developed completely in control conditions. When they reached the adult stage, 139 we created 13 full-sib families per selection line by transferring one virgin male with one virgin 140 female of the same selection line in $15 \mathrm{~mL}$ tubes with $1 \mathrm{~g}$ of medium. After four days, in which the 141 beetles could mate and lay eggs, $9 \mathrm{~g}$ of medium was added to provide food for the developing

142 offspring and each mating pair was transferred to a new vial. We repeated this three times,

143 resulting in four vials per mating pair containing medium and eggs. Immediately after removal of

144 the mating pair, vials of each mating pair were randomly assigned to the four different 145 conditions, resulting in full-sib families split across all conditions. These beetles were transferred 146 to the treatments at the egg stage. As soon as offspring in these vials had reached the pupal 147 stage, males and females (four females and four males per family and condition) were separated 148 and transferred to $15 \mathrm{~mL}$ tubes with $5 \mathrm{~g}$ of medium and remained there until they were used for 149 the fitness assay two weeks later. They developed completely in treatment conditions. We then 
150 assessed their performance in each condition by estimating their fitness to test for adaptation. A

151 virgin male and a virgin female of the same selection line from the same condition but from

152 different families were again placed into a $15 \mathrm{~mL}$ tube with $1 \mathrm{~g}$ medium. After four days, the

153 mating pair was removed. Males and females were transferred to $1 \mathrm{~mL}$ Eppendorf tubes (one

154 individual per tube), immediately frozen in liquid nitrogen and stored at $-80^{\circ} \mathrm{C}$ to use them for

155 gene expression measurements. $9 \mathrm{~g}$ medium was added to the mating tube. After four weeks (in

$156 \mathrm{CT}$ and $\mathrm{H}$ ) or five weeks (D and HD), all offspring had reached the adult stage and were counted.

157 We used the number of adult offspring as an estimate of the fitness of a mating pair.

159 Statistical analysis

160 To test whether selection regime significantly influenced number of offspring produced and test

161 whether 20 generations in the treatments resulted in adaptation, we compared offspring

162 numbers of selection lines in their native condition to CT-lines transferred to the same condition.

163 We applied linear mixed models using the R-packages Ime4 (Bates, Mächler, Bolker, \& Walker,

164 2015), and Imertest (Kuznetsova, Brockhoff, \& Christensen, 2017) and Ismeans (Lenth, 2016) to

165 obtain $p$-values and confidence intervals. We included line and family as random factors,

166 selection and line type (mixed/normal) and their interaction as fixed effects. To test whether the

167 selection regime influenced how lines responded to the treatments, we used a linear mixed

168 model with offspring number in CT and treatment conditions as response variable, condition,

169 selection regime, line type (normal/mixed) and interactions as fixed effects and line, family and

170 interaction between line and condition as random effects. A significant interaction between

171 condition and selection regime indicates a significant effect of the selection regime (evolution)

172 on the response to the conditions (plastic response).

173

$174 \quad$ RNA extraction, library preparation and sequencing 
175208 female beetles (Table 1) p stored at $-80^{\circ} \mathrm{C}$ were homogenized in Tri-Reagent ${ }^{\circledast}$ (Zymo

176 Research, California, USA) using an electric bead mill. RNA was extracted with the RNA Mini Prep

177 kit (Zimo Research, California, USA) following the instructions of the manufacturer. RNA-quality

178 was checked on a TapeStation (Agilent, Waldbronn, Germany) and concentrations were

179 measured with aQubit ${ }^{\circledR}$ Fluorometer (Life Technologies, California, USA). Libraries were created

180 with 500 ng RNA for each individual separately with the LEXOGEN mRNA-Seq Library Kit

181 following the manual (LEXOGEN GmbH, Vienne, Austria). Library quality was checked on a

182 TapeStation (Agilent, Waldbronn, Germany) and concentrations were determined by qPCR.

183 Libraries were diluted to the same molarity and pooled (33-36 libraries per pool). All treatments

184 and selection regimes were randomized during RNA-extraction, library preparation, and

185 sequencing. Single-end sequencing was performed in five runs on the Illumina NextSeq 500

186 (Illumina, Inc, California, USA) using the 75 cycles High Output Kit. After quality control using

187 FastQC (www.bioinformatics.bbsrc.ac.uk/projects/fastqc) reads (adaptors were trimmed and the

188 first 10 bases were hard trimmed, minimum average quality Q10, minimum tail quality 10 ,

189 minimum read length 20) were mapped against the reference genome

190 (ftp://ftp.ensemblgenomes.org/pub/release30/metazoa/gtf/tribolium_castaneum/Tribolium_ca

191 staneum.Tcas3.30.gtf.gz) with STAR v.2.5 (Dobin et al., 2013). We then used FeatureCounts (Liao,

192 Smyth, \& Shi, 2014) to count the number of reads that mapped to each gene in the reference

193 genome. Mapping as well as read counting was performed within the data analysis framework

194 SUSHI (Hatakeyama et al., 2016).

196 Gene expression analysis

197 Gene expression analysis was done in $\mathrm{R}$ ( $\mathrm{R}$ Core Team, 2017). We used the R package edgeR

198 (Robinson, McCarthy, \& Smyth, 2010) for normalizing (method: TMM) expression data to cpm

199 (counts per million) after filtering lowly expressed genes (minimum of one cpm in at least two 
samples). For subsequent differential expression analysis we used the R package limma (Law,

201 Chen, Shi, \& Smyth, 2014; Ritchie et al., 2015). From the differential expression analysis, we obtained the number of differentially expressed genes (DE genes) within lines between conditions (plastic changes, see Figure S1) or between lines of different origins (CT vs. selection)

204 within conditions (evolutionary changes, see Figure S1). The total phenotypic divergence in gene 205 expression between CT and treatments (i.e. total change TC) is the differential expression (log2206 fold change) between CT-lines in CT and selection lines in the treatments (Figure 1A-C and 207 Supporting information Figure S1). The ancestral plasticity $\left(\mathrm{PC}_{\mathrm{CT}}\right)$ is the differential expression of 208 CT-lines between $\mathrm{CT}$ and treatment conditions, while the evolved plasticity $\left(\mathrm{PC}_{\mathrm{Sel}}\right)$ is the same 209 difference measured in selection lines. The evolutionary changes are $\mathrm{EC}_{\mathrm{T}}$ when measured as 210 differential expression between $\mathrm{CT}$ and selection lines in the treatments and $\mathrm{EC}_{\mathrm{CT}}$ when 211 measured in CT (Figure 1). Finally, differences between plastic responses of CT- and selection 212 lines (the interaction between condition and selection regime) give the evolutionary change in 213 plasticity. To partition TC into changes explained by ancestral plasticity $\left(\mathrm{PC}_{\mathrm{CT}}\right)$ and evolutionary 214 changes $\left(E C_{T}\right.$, see Figure $\left.1 A-C\right)$, we calculated the relative contribution of each component to the 215 total. We used the log2-fold change of each transcript to evaluate and compare the magnitude 216 of the plastic and evolutionary changes. When computing log2-fold changes, we accounted for 217 non-independence among individuals from the same line by using the duplicatecorrelation 218 function (Smyth et al. 2005) and added sequencing runs as batch effect. A gene is classified as 219 differentially expressed (DE) with a FDR $\leq 5 \%$ after adjusting for multiple testing (Benjamini and $220 \quad$ Hochberg 1995).

221 In a second step, we used the normalized read counts (cpm, TMM-normalized) corrected for 222 batch effects (sequencing runs) using the removeBatchEffect function in the limma R package 223 (Ritchie et al., 2015). We used these counts to quantify expression levels in in CT and treatments. 
224 This allowed us to conduct an analysis for each selection lines separately as was described by Ho

225 \& Zhang (2018) (details see below).

229 Reaching a new phenotypic optimum can be achieved by changing the mean (intercept of the 230 reaction norm) or by changing the plasticity of a trait (slope of reaction norm) (Figure 1D,E). We

231 were interested in assessing which of these patterns was more prevalent in gene expression 232 evolution. To test for an evolutionary change in plasticity (slope), we first evaluated the similarity 233 in plasticity between CT- and selection lines by calculating the correlation between $\mathrm{PC}_{\mathrm{CT}}$ and $\mathrm{PC}_{\mathrm{Sel}}$

234 (Suppurting information Figure S2A) among all genes, independent of the significance. Second, 235 to test for an evolutionary change in mean expression (intercept), we calculated the correlation 236 between the evolved differences in $\mathrm{CT}\left(\mathrm{EC}_{\mathrm{CT}}\right)$ and in treatment conditions $\left(\mathrm{EC}_{\mathrm{T}}\right)$. A significant 237 correlation indicates that the overall mean has changed (Figure S2A). Further, for all genes with 238 significant $E C_{T}$, we quantified how much of this difference could be explained by a shift in the 239 mean or by a change in plasticity. A shift in the mean corresponds to $\mathrm{EC}_{\mathrm{CT}}$, whereas the 240 remaining difference $\left(\mathrm{EC}_{\mathrm{T}-\mathrm{EC}}-\mathrm{C}_{\mathrm{CT}}\right.$ ) gives the evolutionary changes in plasticity (see also Stoks et al.

241 2016). Relative contributions of each component were obtained by dividing them by the total $242 \quad \mathrm{EC}_{\mathrm{T}}$.

244 To test for significant effects of the selection regime on differential expression (i.e., on number 245 of DE genes, log2-fold change, and correlations), we used a permutation test. We randomly 246 assigned samples and their transcriptomes to either CT selection or treatment (D, H, HD) 247 selection (number of samples for each selection was not changed) and repeated the DE analysis. 248 We kept the original assignment to lines and conditions and repeated the DE analysis for each 
249 permuted data set. Observed values (e.g. number of DE genes, correlations) were considered

250 significant if higher than the most extreme $5 \%$ of the distribution calculated from permutations.

254 To infer the relationship between ancestral plasticity and evolution, we compared the direction

255 of $\mathrm{PC}_{\mathrm{CT}}$ to the direction of $\mathrm{EC}_{\mathrm{T}}$. Evolution may reinforce the plastic response when $\mathrm{PC}_{\mathrm{CT}}$ is in the 256 same direction as $\mathrm{EC}_{\mathrm{T}}$ (Figure 1B). If $\mathrm{EC}_{\mathrm{T}}$ is in opposite direction, it reverses $\mathrm{PC}_{\mathrm{CT}}$ (Figure $\left.1 \mathrm{~A}\right)$. To 257 test which of these patterns was more prevalent, we followed Ho and Zhang 2018 (Ho \& Zhang, 258 2018). Expression levels (cpm) of CT-Lines in CT conditions represented the original stage (Lo), 259 CT-Lines in treatment the plastic stage $(L p)$, and selection lines in their respective condition the 260 adaptive stage ( $L a)$ (Figure 2). For subsequent analysis we used genes with appreciable $\mathrm{PC}_{\mathrm{CT}}(\mid L p$ $261-L o \mid>0.2 L o)$ and $\mathrm{EC}_{\mathrm{T}}(|L a-L p|>0.2 L o)$ (Ho \& Zhang, 2018) and calculated the proportion of 262 plastic genes with reinforced $\left(\mathrm{TC}>\mathrm{PC}_{\mathrm{CT}}\right)$ or reversed $\left(\mathrm{TC}<\mathrm{PC}_{\mathrm{CT}}\right)$ changes.

263 An alternative classification of plastic responses is to assess whether $\mathrm{PC}_{\mathrm{CT}}$ brings expression 264 levels closer to the new optimum ( $L a)$. In this case, the total change $\mathrm{TC}$ would be larger than $\mathrm{EC}_{\mathrm{T}}$

265 (Figure $2 \mathrm{C}$ ). Such a pattern can occur even if $\mathrm{PC}_{\mathrm{CT}}$ and $\mathrm{EC}_{\mathrm{T}}$ are in opposite directions and classified 266 as reversion (Figure $2 \mathrm{C}$ ), but may indicate that ancestral plasticity was beneficial. A $\mathrm{PC}_{\mathrm{CT}}$ that 267 moves $L p$ further away from $L a$ is strongly indicative of maladaptive plasticity.

268 To confirm that our results were not sensitive to the applied cutoff ( $20 \%$ Lo), we repeated the 269 analysis with a cutoff of 50\% Lo and without any cutoff (Supporting information, Figure S3.1 A270 H). Recently, it was pointed out (Mallard et al. 2018; Ho and Zhang 2019) that an excess of 271 reversions relative to reinforcements is expected to be observed due to a statistical artefact that 272 cannot be completely removed by permutation tests. We therefore applied an additional 
273 parametric bootstrapping test as proposed by Ho and Zhang (Ho and Zhang 2019), to compare

274 proportion of reversions and reinforcements (Supporting information, Figure S3.1 J-L).

275 To better understand the relationship between the within-line proportions of reversed genes

276 and proportions of genes with La closer to Lo with adaptation, we calculated the Spearman

277 correlation between the proportions of reversed expression changes (or La closer to Lo

278 respectively) and mean offspring number in seven selection lines in HD. We focused on HD

279 because it was the most extreme environment with the strongest decline in offspring number.

280 To test for significance, we used permutations: Mean offspring numbers were randomly assigned

281 to lines and correlation was calculated again. Proportion of permutations with a correlation

282 coefficient exceeding the observed value gave the respective $P$-value. 


\section{$284 \quad$ Results}

\section{Fitness assay showed evolutionary adaptation}

286 We found that selection lines had a higher offspring number in their native condition compared to CT-lines (Dry: $\mathrm{F}_{1,14}=9.20, p=0.009 ;$ Hot: $\mathrm{F}_{1,16}=4.78, p=0.044$; Hot-Dry: $\mathrm{F}_{1,16}=23.51, p=$

288 1.786E-04), confirming that adaptation had occurred (Figure 2). In contrast to treatment 289 conditions, there was no difference in offspring number between CT- and selection lines under 290 CT conditions (CT-lines: 64.89 [61.49, 68.29]; D-lines: 65.76 [61.26, 70.25], H-lines: 67.41 [63.76, 291 71.06], HD-lines: 65.56 [62.17, 68.94]) (Figure 2). Using three additional mixed models, we 292 compared how lines from different selection regimes responded to treatments. We found 293 significant negative effects for all stress treatments ( $D: F_{1,23}=45.37, p=6.85 E-07 ; H: F_{1,28}=$ $294507.68, p<2.200 \mathrm{E}-16 ; \mathrm{HD}: \mathrm{F}_{1,28}=553.06, p<2.200 \mathrm{E}-16$ ) (Figure 2). Interaction between selection 295 and treatments, i.e. whether the response to the treatment was different depending on 296 selection regime, was significant for HD-lines $\left(F_{1,28}=9.39, p=4.754 \mathrm{E}-03\right)$ and for $\mathrm{D}\left(\mathrm{F}_{1,23}=4.32, p\right.$ $297=0.049)$, but not for $\mathrm{H}\left(\mathrm{F}_{1,28}=0.57, p=0.455\right)$. Interaction between selection regime, treatment 298 and line type (normal/mixed) was not significant in any treatment indicating that mixing lines did 299 not have an effect on adaptation. ANOVA tables as well as results of the linear mixed models are $300 \quad$ in supporting information S4.

$302 \quad$ Evolution of gene expression

303 We found many more genes with expression divergence relative to Control (TC) than genes with 304 evolved changes in the treatments $\left(E C_{T}\right)$ or in control conditions $\left(E C_{C T}\right)$ (Table 2). Increased 305 temperature caused expression divergence in a larger number of genes than reduced humidity 306 (Table 2). Most DE genes with significant TC also had significant ancestral plasticity $\mathrm{PC}_{\mathrm{CT}}$ (Dry: 33.9\%; Hot: $72.9 \%$, Hot-Dry: $82.2 \%$ ), but only a minority showed significant evolutionary change 
$308 \mathrm{EC}_{\mathrm{T}}$ (Dry: 4.9\%; Hot: 1.1\%; Hot-Dry: 1.0\%, see also Supporting information Figure S3.2). About

309 half of the DE genes with $\mathrm{EC}_{\mathrm{T}}$ in Hot and Hot-Dry then did not diverge significantly from their

310 ancestral expression levels in Control (TC 0). Overall, TC had a larger log2-fold change

311 contribution from plastic changes $\left(\mathrm{PC}_{\mathrm{CT}}\right)$ than evolved changes in Hot and Hot-Dry (median

312 proportions $\mathrm{PC}_{\mathrm{CT}} / \mathrm{TC}$; Hot: $71.47 \%$; Hot-Dry: $\left.86.30 \%\right)$, but not in Dry (42.90\%). Many genes had

$313 \quad \mathrm{PC}_{\mathrm{CT}}>\mathrm{TC}$, especially in $\mathrm{HD}$ (Figure $3 \mathrm{~A}$ ). In these cases, $\mathrm{EC}_{\mathrm{T}}$ was negative and reduced TC.

$314 \quad$ Evolution of reaction norms

315 We were then interested to examine whether $\mathrm{EC}_{\mathrm{T}}$ were due to a change in mean expression

316 (shift in the intercept of reaction norms) or to a change in plasticity (different slopes of reaction

317 norms, see Figure 3). First, we found a significant correlation between $\mathrm{EC}_{\mathrm{CT}}$ and $\mathrm{EC}_{\mathrm{T}}$, in all three

318 treatments (permutation tests: $P<0.0001$ ) (Table 3, Supporting information Figure S2). This

319 indicates that for a majority of genes, evolution in the past 20 generations affected their mean

320 expression levels by shifting the intercept of reaction norms (see Figure 1D). Second, we also

321 found a highly positive correlation between $\mathrm{PC}_{\mathrm{CT}}$ and $\mathrm{PC}_{\text {Sel }}$ (Figure Supporting information $\mathrm{S} 2 \mathrm{~A}$ ),

322 indicating that plasticity was mainly preserved during adaptation to new conditions. A

323 permutation test showed that this correlation was not significantly different from correlations

324 obtained from permuted datasets (Table 3), where samples were randomly assigned to selection

325 regimes, suggesting that the treatments did not have specific effects on plasticity. When we

326 quantified the relative contributions of changes in the mean versus changes in plasticity to $\mathrm{EC}_{\mathrm{T}}$,

327 we found that evolution of the intercept explained more evolutionary divergence than evolution

328 of the slope of reaction norms, especially in Dry (Figure 3B).

329 Evolution of plasticity in DE genes

330 We found only five genes with significantly evolved plasticity in the DE analysis, after correcting

331 for multiple testing. Although plasticity seemed to be preserved overall, we found evidence that 
332 it evolved among DE genes. The DE analysis revealed a reduced number of plastic genes in

333 selection lines compared to plastic genes in CT-lines (Table 2), although the difference was only

334 significant in $\mathrm{HD}$ (Table 3). It further showed that ancestral plasticity $\mathrm{PC}_{\mathrm{CT}}$ was larger than evolved 335 plasticity $\mathrm{PC}_{\text {Sel, }}$ in $\mathrm{D}$ and $\mathrm{HD}$ (Table 3), indicating a reduction of the magnitude of plastic 336 responses after adaptation. We found the same outcome when focusing on genes with 337 significant $\mathrm{EC}_{\mathrm{T}}$, in HD (Mann-Whitney $\mathrm{U}$ test: $\mathrm{U}=2133, P=2.102 \mathrm{E}-04$; median: $\mathrm{CT}$-lines: $0.25, \mathrm{HD}$ 338 lines: 0.13$)$, but not in $\mathrm{D}(\mathrm{U}=144, P=0.584$; median: $\mathrm{CT}$-lines: 0.10 , $\mathrm{D}$-lines: 0.14$)$ or $\mathrm{H}(\mathrm{U}=386$, $339 P=0.158$; median: CT-lines: 0.21, H-lines: 0.12) (Figure 4).

\section{$341 \quad$ Effect of plasticity on evolutionary responses}

342 We could not find evidence that plasticity in general impeded evolution. In the DE analysis genes 343 with a significant ancestral plastic response were overrepresented among the evolved DE genes

344 in $\mathrm{H}$ and $\mathrm{HD}(\mathrm{H}: \chi 2=4.51, \mathrm{df}=1, P=3.38 \mathrm{E}-02 ; \mathrm{HD}: \chi 2=9.71, \mathrm{df}=1, P=1.84 \mathrm{E}-03)$, but not in $\mathrm{D}(P$ $345=0.113$ Fisher's exact test). Furthermore, when we analysed each selection line separately and 346 compared their expression levels to $\mathrm{CT}$-lines, the proportion of genes exhibiting substantial $\mathrm{PC}_{\mathrm{CT}}$ $347(|L p-L o|>0.2 L o)$ were also overrepresented among evolved genes with $\mathrm{EC}_{\mathrm{T}}(|L a-L p|>0.2 L o)$ $348 \quad$ (Table S5.1).

349 Maladaptive plastic changes may be reverted by evolutionary changes when the plastic response 350 overshoots the optimum expression level in a new environment. Among the genes with 351 significant $\mathrm{EC}_{\mathrm{T}}$ in the $\mathrm{DE}$ analysis, we found that $\mathrm{PC}_{\mathrm{CT}}$ was generally in opposite direction (Figure 352 4). Enrichment tests revealed that genes with positive $E C_{T}$ in the $D E$ analysis were 353 overrepresented among genes with negative $\mathrm{PC}_{\mathrm{CT}}$ in $\mathrm{H}$ and $\mathrm{HD}$ (Fisher's exact test $\mathrm{D}: \mathrm{P}=0.076 ; \mathrm{H}$ : $354 P=0.002 ; \mathrm{HD}: P=9.26 \mathrm{e}-06)$, and genes with negative $\mathrm{EC}_{\mathrm{T}}$ were overrepresented among genes 355 with positive $\mathrm{PC}_{\mathrm{CT}}$, in $\mathrm{H}$ and in $\mathrm{HD}$ as well (Fisher's exact test $\mathrm{D}: P=0.073 ; \mathrm{H}: P=0.021 ; \mathrm{HD}: P=$ 
0.049)(Figure 4). In almost all genes that showed significant $\mathrm{EC}_{\mathrm{T}}$ and $\mathrm{PC}_{\mathrm{CT}}$, the responses were in opposite direction (Dry: 2 out of 2 genes; Hot: 12 out of 13; Hot-Dry: 33 out of 34). We also found negative correlations between $\mathrm{PC}_{\mathrm{CT}}$ and $\mathrm{EC}_{\mathrm{T}}$ in all treatments, although significant only in $\mathrm{H}$ and HD, but not in D (Supporting information, Figure S3.3).

360 When we considered mean expression levels per line with $\mathrm{PC}_{\mathrm{CT}}>20 \% \mathrm{Lo}$ and $\mathrm{EC}_{\mathrm{T}}>20 \% \mathrm{LO}$, we 361 obtained similar results as in the DE analysis. Reinforcements were present but less frequent 362 than reversions in all treatments (proportions of plastic responses with reversion/reinforcement: 363 Dry: $48.5 \pm 1.0 \% / 34.3 \pm 0.8 \%, P<1.151 \mathrm{e}-07$ (binomial test); Hot: $44.6 \pm 0.9 \% / 28.8 \pm 1.2 \%, P=$ 364 4.369E-13; Hot-Dry: $43.6 / 27.1 P<2.2 \mathrm{e}-16$; Figure 5A). The reversed genes represented $34.1 \pm$ $365 \quad 0.8 \%$ (Dry), $40.0 \pm 0.9 \%$ (Hot), and $42.96 \pm 2.1 \%$ (Hot-Dry) of the evolved genes. In contrast, 24.1 $366 \pm 0.6 \%$ (Dry), $25.8 \pm 1.1 \%$ (Hot), $26.80 \pm 1.6 \%$ (Hot-Dry) of evolved genes showed reinforcement.

367 Different cut-off values did not influence these outcomes (Figure S3.1). Parametric bootstrapping 368 as an additional test (Ho \& Zhang, 2019), confirmed our results (Supporting information, Figure 369 S3.1). We also found that a large proportion of ancestrally plastic genes had La closer to Lo than 370 to $L p$ (Dry: $45.4 \pm 1.1 \%$; Hot: $41.2 \pm 1.2 \%$; Hot-Dry: $40.7 \pm 2.5 \%$ )(Figure $5 B$ ). Finally, substantial 371 proportions of genes with $\mathrm{EC}_{\mathrm{T}}$ did not show $\mathrm{PC}_{\mathrm{CT}}$ (Dry: $41.8 \pm 0.7 \%$, Hot: $34.6 \pm 0.8 \%$, Hot-Dry: $372 \quad 30.3 \pm 1.0 \%)$.

373 In case of genetic assimilation, i.e. loss of plasticity in selection line and continuous expression of 374 the induced phenotype, evolutionary differences would not be visible in treatment condition. 375 We calculated the mean expression levels of selection lines in Control and defined genes as 376 showing assimilation if they met three criteria: no $\mathrm{PC}_{\mathrm{sel}}$ (absolute difference between selection 377 lines in $C T$ and in treatment $<20 \% \angle o$ ), $P C_{C T}>20 \% L O$, and $L p \sim L a$ (absolute difference $<20 \% \angle a$ ). 378 The proportion of $\mathrm{PC}_{\text {Ст }}$ genes showing genetic assimilation was small in all conditions (Dry: $6.8 \%$, 379 Hot: $10.3 \%$, Hot-Dry: $10.3 \%$; Supporting information Table S5.2). 

evolved lines, we tested for an association between within-line proportion of reversed or

384 reinforced plastic responses and the average fitness of the lines in the HD treatment. We found 385 that lines with a higher proportion of reversions had a higher average offspring number 386 (correlation: $0.82, P=0.012$, Figure $6 \mathrm{~A}$ ) and we found a negative but non-significant correlation 387 between fitness and reinforcements (correlation: $-0.43, P=0.85$ ). When we tested for an 388 association between fitness and proportion of ancestrally plastic genes with La closer to Lo, we 389 also found a positive correlation (correlation: $0.86, P=0.006$, Figure $6 \mathrm{~B}$ ). Overall, better adapted 390 lines (higher fitness in HD) showed a higher proportion of reversed ancestral plasticity and these 391 plastic genes were more similar to the original expression levels of CT-lines in CT. Performing the 392 analysis with gene expression data in $H$ and $D$ provided similar correlations, although not 393 significant because of lower sample sizes (Supporting information, Table S5.3).

\section{Discussion}

396 We studied plastic and evolved responses in gene expression of $T$. castaneum in response to 397 three new environmental conditions (Dry, Hot, Hot-Dry). After 20 generations of experimental 398 evolution, we were able to detect adaptation and found significant evolutionary changes in 399 expression levels. Comparing evolutionary changes with ancestral plastic responses showed that 400 a reversion of plasticity was most frequent (> $95 \%$ genes with significant plastic changes in DE 401 analysis; > $40 \%$ of genes with substantial plastic changes, i.e. changes higher than $20 \%$ of 402 ancestral levels). The number of genes where ancestral plasticity was reinforced by evolution 403 was significantly smaller (DE analysis: $<5 \%, 27-34 \%$ of genes with substantial plastic changes) 404 and a smaller proportion of genes (7-10\% of substantially plastic genes) showed expression 
405 patterns consistent with genetic assimilation. A high proportion of the originally plastic genes

406 evolved to expression levels that were closer to control levels than to ancestrally plastic levels.

407 Although the proportion of non-reversed plastic genes was still high, positive associations

408 between fitness and proportion of reversions, and compensated plasticity (expression levels

409 closer to control levels) respectively, suggest that ancestral plasticity was maladaptive for a

410 majority of responding genes. Although plasticity showed a high degree of preservation in terms

411 of number of responding genes and direction of the response, we found evidence that selection

412 lines evolved a reduced plasticity and thus partly compensated the maladaptive ancestral

413 response. We were further able to show a positive association between the proportion of

414 reversed plastic responses and adaptation (mean fitness per line) in the most stressful treatment

415 Hot-Dry.

416

$417 \quad$ Maladaptive plasticity

418 Different patterns describing the relationship between plastic and evolved changes in gene

419 expression have been documented. It was suggested that plasticity might help populations to

420 persist after environmental change, or to colonize new habitats by bringing phenotypes closer to

421 the new optimum. Studies found support for this hypothesis by showing that plastic responses of

422 non-adapted individuals diminished differences to native populations (Lohman, Stutz, \& Bolnick,

423 2017; Mäkinen, Papakostas, Vøllestad, Leder, \& Primmer, 2016). Adaptive plasticity can also be

424 indicated when plastic and evolutionary responses are in the same direction ( $\mathrm{Li}$, Li, Song, Wang,

425 \& Zhang, 2017), or when plasticity is higher in adapted populations (Hasan et al., 2017; McCairns

426 \& Bernatchez, 2009), suggesting that most plastic individuals were favored by selection.

427 However, there are also examples for the reversed pattern suggesting that plasticity was

428 maladaptive. In wild populations of Fundulus heteroclitus, evolved changes to different

429 temperatures were opposite to plastic responses of the ancestral population (Dayan et al., 
430 2015). Rhagoletis flies shifting to a new host fruits showed evolutionary responses opposite to

431 plasticity of non-adapted species (Ragland et al., 2015). Experimental evolution studies found

432 countergradient evolution in Drosophila adapting to different diets (Huang \& Agrawal, 2016;

433 Yampolsky et al., 2012) and in guppies adapting to low predation environments (Ghalambor et

434 al., 2015). A comparative study (Ho \& Zhang, 2018) analyzing data of multiple experimental

435 evolution suggested that reversions of gene expression changes might be a general pattern

436 during adaptation.

437 Our study fits with these previous observations. We found a higher proportion of reversions than

438 reinforcements in all conditions indicating mostly maladaptive plasticity. An alternative

439 explanation for the prevalence of reversions without maladaptive plastic responses would be

440 that CT-lines exhibited a response in the right direction, but overshot an optimum expression

441 level (Figure 1C). Fine-tuning during long-term adaptation could then lead to a partial reversion

442 of the plastic response. We took this possibility into account by not only focusing on a reversion

443 of plasticity, but also testing whether plastic changes brought expression levels closer to the

444 adapted stage $L a$ than to original stage $L o$. If, in contrast, we see that $L a$ is closer to the original

445 level (CT lines in CT conditions), it indicates that plastic responses were maladaptive since they

446 moved expression levels further away from the new optimum and became compensated during

447 evolution (see Figure 1C). We found that adapted lines showed a high proportion of expression

448 levels closer to their ancestral level $L o$ than to $L p$. We could further show that this proportion is

449 positively associated with higher fitness per selection line. We also found strong positive

450 associations of within-line proportions of reversions with mean reproductive output, indicating a

451 possible fitness advantage to reversions.

452 There was still a large proportion of plastic genes that did not show reversion. They are either

453 close to the levels of adapted lines, or showed reinforcement. The first case might indicate that

454 plasticity prevented evolution by matching the new optimum. Reinforced plastic changes could 
455 be examples of adaptive plasticity. However, correlation between proportion of reinforcements

456 and fitness was not significant and negative. The observed positive correlation between

457 proportion of reversed plastic genes and mean fitness per line in HD rather suggests that

458 reversions were favoured during adaptations. Reversions might become more pronounced after

459 more generations, once evolution had sufficient time to further reverse maladaptive ancestral

460 plastic responses. Our selection lines still show a strong reduction in offspring number compared

461 to control levels suggesting further potential to adapt.

\section{$463 \quad$ Evolution of reaction norms}

464 The ancestral maladaptive plasticity can be compensated by shifts in the intercept or changes in

465 the slope of reaction norms. Both are not mutually exclusive and can occur together in the same

466 trait. We were aiming to quantify their relative importance for evolutionary responses in

467 transcriptomes.

469 Gene expression studies so far provided mixed results regarding the evolution of plasticity.

470 Drosophila populations adapted to different temperatures showed local adaptation, but there

471 was no evidence for evolution of thermal reaction norms of different transcripts and changes

472 affected mainly expression mean (Clemson, Sgrò, \& Telonis-Scott, 2016). Experimental evolution

473 studying Drosophila under variable diets found no significant changes in plasticity (Yampolsky et

474 al., 2012) or less than expected (Huang \& Agrawal, 2016). In contrast, other studies found

475 differences in temperature responses between tropical and temperate Drosophila populations

476 (Levine, Eckert, \& Begun, 2011; von Heckel et al., 2016). Other examples for differences in genes

477 expression plasticity between adapted and non-adapted populations include temperature

478 (Morris et al., 2014) and salinity (Gibbons, Metzger, Healy, \& Schulte, 2017; McCairns \&

479 Bernatchez, 2009) responses of marine and freshwater sticklebacks, temperature response of 
480 killifish populations from different latitudes, as well as plastic responses to toxic hydrogen

481 sulphide $\left(\mathrm{H}_{2} \mathrm{~S}\right)$ of fish population from $\mathrm{H}_{2} \mathrm{~S}$ rich springs versus non-toxic springs (Passow et al.,

482 2017). There was no consistent pattern regarding the direction in which plasticity evolves: In

483 some cases adapted population showed an increase in plasticity (Morris et al., 2014), in other

484 cases plasticity was reduced (Huang \& Agrawal, 2016; Ragland et al., 2015; von Heckel et al.,

485 2016) or reduction and enhancement of plasticity were equally frequent (Gibbons et al., 2017;

486 Yampolsky et al., 2014). Overall, there is evidence in multiple species that expression plasticity of

487 some genes can evolve. However, even in some of these studies reporting evolved plasticity

488 (Dayan et al., 2015; Gibbons et al., 2017; Morris et al., 2014) the number of transcripts with

489 significant changes in the mean was much higher than transcripts with changed plasticity and

$490 \quad$ large parts of the plastic responses showed a high degree of preservation.

492 In accordance with these previous findings we found that changes in the mean contributed more

493 to the observed expression differences in the treatments than changes in plasticity. A possible

494 reason might be that genetic variation in mean expression was higher than genetic variation in

495 plasticity. In addition, we did not select for changes in plasticity directly since the conditions in

496 the treatments were constant. Selection was therefore on expression levels in the treatment and

497 only indirectly on plasticity. Plasticity could evolve if mean expression levels were genetically

498 correlated with plasticity. Although we did not directly test for such correlations, we found

499 evidence for evolution of plasticity of the DE genes, that is of the genes with significant changes

500 in expression level between CT and treatment conditions. Although plastic responses showed a

501 high degree of preservation in terms of affected genes and direction, we found evidence for

502 evolutionary changes in the magnitude of plastic responses, i.e. the slope of the reaction norm.

503

504 
506 New stressors might disturb homeostasis resulting in inappropriate responses and long-term

507 adaptation therefore restores ancestral phenotypes by genetic changes, referred to as genetic

508 compensation (Grether, 2005) or counter-gradient variation (Conover et al., 2009). However, in

509 our study we applied relatively mild stressor treatments, i.e. individuals were able to survive and

510 reproduce. Drought and heat are also stressors, which T. castaneum had experienced in the past

511 (Sokoloff, 1972), so there had probably been selection on plastic responses to be beneficial.

512 However, plastic responses might be optimized for a short-term exposure: Allocation of

513 resources from reproduction to protection might increase survival probability and allow

514 individuals to continue reproduction as soon as the stress has disappeared, but this response

515 becomes maladaptive during continuous exposure and should therefore be under negative

516 selection. Expression of stress related genes is in general accompanied by a down-regulation of

517 genes involved in growth and reproduction due to an allocation of resources (Schwenke, Lazzaro,

518 \& Wolfner, 2016; Sokolova, 2013). A well-studied example are heat shock proteins (hsp). Hsp are

519 well known for their protective function and to be crucial for survival (Feder \& Hoffman, 1999),

520 but it was also shown that their expression comes at a cost (Feder et al., 1998; Sørensen,

521 Kristensen, \& Loeschcke, 2003). Accordingly, it was often found that hsp expression in

522 populations adapted to warmer climates is lower compared to non-adapted populations

523 (Fangue, Hofmeister, \& Schulte, 2006; Narum \& Campbell, 2015; J G Sørensen, Dahlgaard, \&

524 Loeschcke, 2001). In general, other protection mechanisms independent of ancestral plasticity

525 may arise during long-term adaptation (e.g. enzymes, which are more stable at high

526 temperature) and make the costly stress response expendable.

528 An alternative explanation for the reduced plasticity in adapted lines is that the signal 529 responsible for eliciting the plastic responses is based on any kind of damage (e.g. deformations 
530 in macromolecules, membrane lipids, proteins, and DNA) caused by heat or stress in general

531 (Kültz, 2005). Higher resistance in adapted lines might shift the inducing thresholds, i.e. the

532 temperature when damages occur and stress response is induced (Sikkink, Reynolds, Ituarte,

533 Cresko, \& Phillips, 2014) above the levels we applied in the treatments.

535 Interestingly, we found no differences in fitness between lines from different selection regimes 536 under CT conditions (Figure 2). We could detect some genes with different expression levels in 537 the treatments and found in general a correlated change in expression levels in control 538 conditions, but this did not affect offspring number. It indicates a lack of fitness trade-offs, 539 where alleles providing a fitness advantage in one environment (treatment) are detrimental in 540 another (CT). Together with the observation that selection lines evolved to bring expression 541 closer to ancestral CT-levels, it suggests that for many genes the optimal expression level is not 542 different between conditions. They might be involved in processes important for maintenance 543 and reproduction. Under stress, limited resources have to be invested into protection, that are 544 then not available for reproduction (Sokolova, 2013). Long-term adaptation should then work to 545 restore control levels that are likely to be optimized for highest reproductive output and to 546 reduce costly stress responses resulting in improved canalization of traits associated with fitness

547 (Stearns \& Kawecki, 2006). Canalization, i.e. robustness against environmental variation, was 548 found previously in gene expression adaptation (Levine et al., 2011; Shaw et al., 2014; von 549 Heckel et al., 2016). Genetic differences between control and selection lines that are responsible 550 for adaptation to the treatments did not have an effect in control conditions and thus represent 551 cryptic genetic variation (Gibson \& Dworkin, 2004). They might either concern genes that are not 552 expressed in control conditions, or represent changes neutral under control conditions. 
556 The number of genes with significant plastic changes in the DE analysis was much higher compared to genes showing evolutionary changes. One possible explanation would be that adaptive plasticity prevented evolution. If the plastic responses matched the optimum, no

559 genetic changes in the selection lines are expected to occur. However, when we analysed each

560 line separately and considered a gene as evolved if the mean difference between $L a$ and $L p$ was

561 more than $20 \%$ of the $L o$, we found approximately the same number of genes with $\mathrm{EC}_{\mathrm{T}}$ and $\mathrm{PC}_{\mathrm{CT}}$

562 (Supporting information Table S5.1).

563 In the DE analysis in limma we did not analyse each line separately but treated them as biological

564 replicates. Since lines were split across conditions comparisons between conditions, i.e. plastic

565 changes, can be made within lines. They should thus be more precise and statistical power

566 should be higher than comparisons between selection regimes, i.e. evolved changes, that have

567 to be made between lines. Differences between lines from the same selection regime lower the

568 ability to obtain significant evolutionary changes. These differences can arise from genetic drift.

569 Since our population size was relatively small (120 individuals per line) this might have been an

570 important factor. Another explanation is that lines from the same selection regime differed how

571 exactly they improved their fitness in the respective treatment. Since fitness is a highly polygenic

572 trait, the genes contributing to a fitness increase may not be the same in different lines (see

573 Barghi et al., 2019). For the most extreme treatment HD, where we sequenced seven lines, we

574 further found considerable differences in fitness between the lines, suggesting that not all of

575 them were at the same stage of adaptation. It is therefore not surprising that expression levels

576 did not evolve in the same way among lines.

577 The DE analysis in limma requires that a gene shows similar changes in all replicate lines, and is

578 therefore more conservative. If the main interest of a study is to identify promising candidate

579 genes for future more detailed analyses it is the appropriate approach to keep FDR as low as 
580 possible. In contrast, if the focus is more on general patterns, a less stringent analysis using mean

581 expression levels can give us a more complete picture. Since genetic drift is random it cannot 582 explain the observed excess of reversions over reinforcements.

584 Although we demonstrated that gene expression changed during evolution, it is not clear 585 whether these changes are the cause of an increased fitness in these conditions or whether they 586 are rather the consequence of adaptation and being less stressed. One disadvantage in studying 587 whole transcriptomes is that not all responding genes might be of functional importance but are 588 correlated to other adaptive changes. High intercorrelations within the transcriptome (Ayroles et 589 al., 2009; McGraw et al., 2011) might lead to correlated responses in many other genes. 590 Furthermore, observed evolutionary changes might be caused by indirect selection and other 591 mechanisms, e.g. changes in protein structure of enzymes, were responsible for adaptation of 592 selection lines. Future studies that manipulate expression and test for correlated changes in 593 offspring are needed to confirm adaptive value of expression changes.

596 We found that genes with the strongest plastic responses showed evolutionary changes in 597 opposite direction suggesting that ancestral plasticity was maladaptive for long-term adaptation.

598 In the most stressful treatment, selection lines with higher fitness show a higher proportion of 599 reversions and a higher proportion of originally plastic genes that are closer to ancestral 600 expression levels. Differences between adapted lines and CT-lines in the treatment were mainly 601 due to a change in mean expression (i.e. shift in the intercept of reaction norms), while plasticity 602 was preserved in terms of affected genes and direction of change. However, we found that a 603 part of the differences in the treatments can be explained by a reduction in the magnitude of 604 plasticity in adapted lines. Our results add to growing evidence that plasticity and evolution are 
605 often in opposite direction and maladaptive plastic responses might increase strength of

606 selection. In contrast to previous studies, including fitness data allowed us to give evidence for

607 adaptation and in the most stressful condition we were able to show an association between

608 reversion of plasticity and adaptation. Similar results in all three stress treatments indicated that

609 these findings may represent a general pattern of gene expression adaptation.

610

611 Data Accessibility

612 RNA-seq reads will be available at GEO.

613 Fitness data will be available at Dryad.

614

615 Author Contribution

616 FG and ELK designed experiment. ELK conducted experiment, laboratory work and analysed the

617 data. FG and ELK wrote the manuscript.

618

619 Acknowledgements

620 This work was supported by the Swiss National Science Foundation, grants PP00P3_1144846 and

621 PP00P3_176965 to FG. We thank Sonja Sbilordo, Sara Meier, Cilgia Lippuner, Tim Schoch and Tim

622 Emmenegger for helping during the fitness assay, Elke Karaus, Martina Berchtold, Encarnación

623 Lozano for RNA extraction and quality control, Lucy Poveda and Maria Domenica Moccia for

624 advice in library preparation and sequencing, Lennart Opitz for helping to process the RNA-seq

625 data.

626 


\section{A}

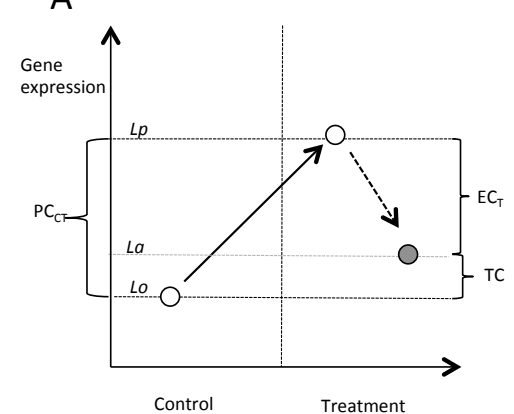

D

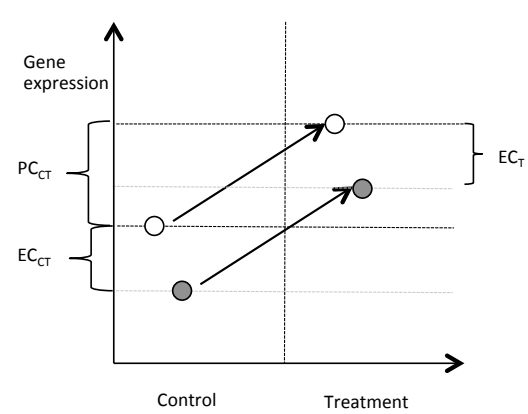

B

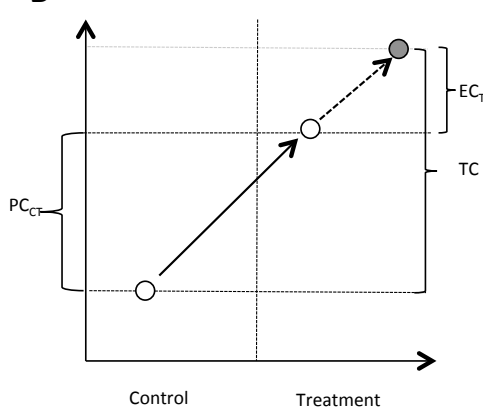

E

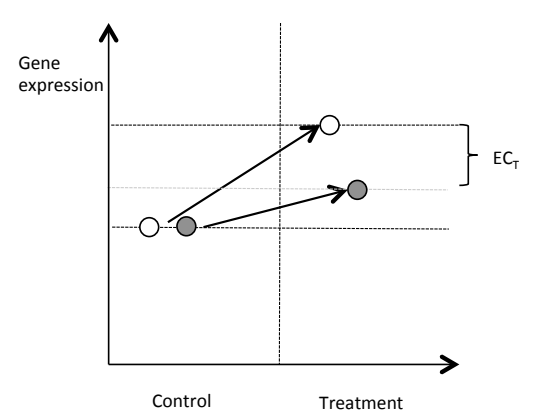

Figure 1: Possible relations between plastic change PC and evolutionary change EC. Gene expression levels of control stage Lp. Expression levels of selection lines (grey) in the treatment give the adapted stage La Arrows indicate the direction of PC (dashed line) and EC (solid line). PC can be in opposite direction to EC (reversion (A)) or it can be in the same direction (reinforcement (B)). However, even if PC and EC are opposite to each other, PC can bring expression levels closer to levels of the adapted lines. In this case, the total change TC, (difference between expression levels of control lines in control conditions and selection lines in treatment) is larger than EC (C). During adaptation, lines could have reached the optimum by either changing mean expression, i.e. shift in the intercept of the reaction norm (D) or by changing their plasticity, i.e. the slope of the reaction norm (E). In case of a change in the mean, plastic changes of Control lines $\mathrm{PC}_{\mathrm{CT}}$ and selection lines $\mathrm{PC}_{\mathrm{Sel}}$ as well as observed evolutionary change $\mathrm{EC}_{\mathrm{T}}$ in treatment and in Control $\mathrm{EC}_{\mathrm{CT}}$ would be highly correlated because reaction norms (red arrows) remain parallel (D). If observed $\mathrm{EC}_{\mathrm{T}}$ in treatment is due to a change in plasticity only, no correlation between $\mathrm{EC}_{\mathrm{T}}$ and $\mathrm{EC}_{\mathrm{CT}}$ should exist, and the correlation between $\mathrm{PC}_{\mathrm{Sel}}$ and $\mathrm{PC}_{\mathrm{CT}}$ should be small (E).
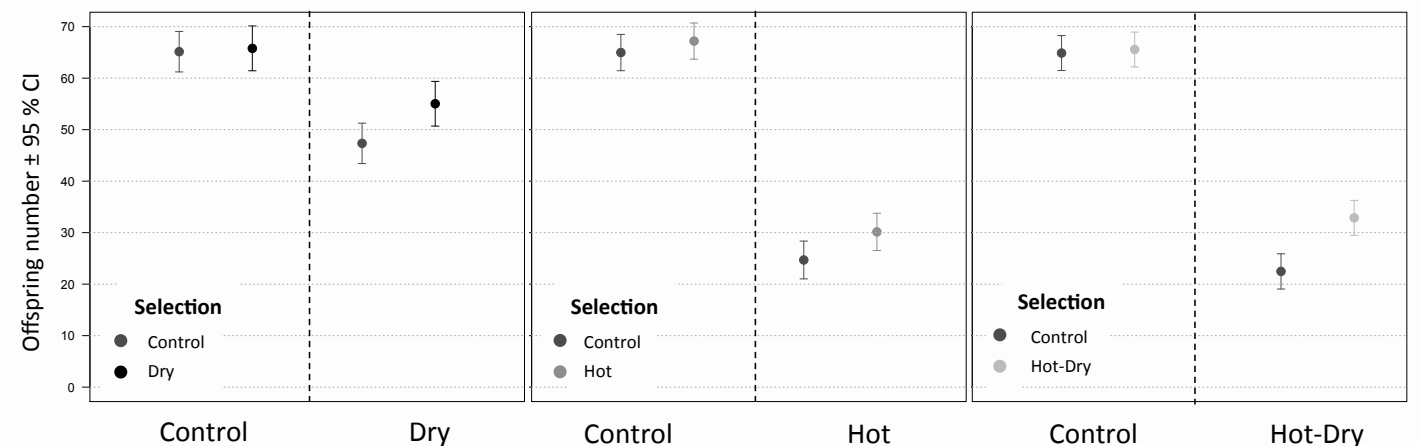

Figure 2: Offspring number of Control and selection lines under different conditions. Selection lines could adapt for 20 

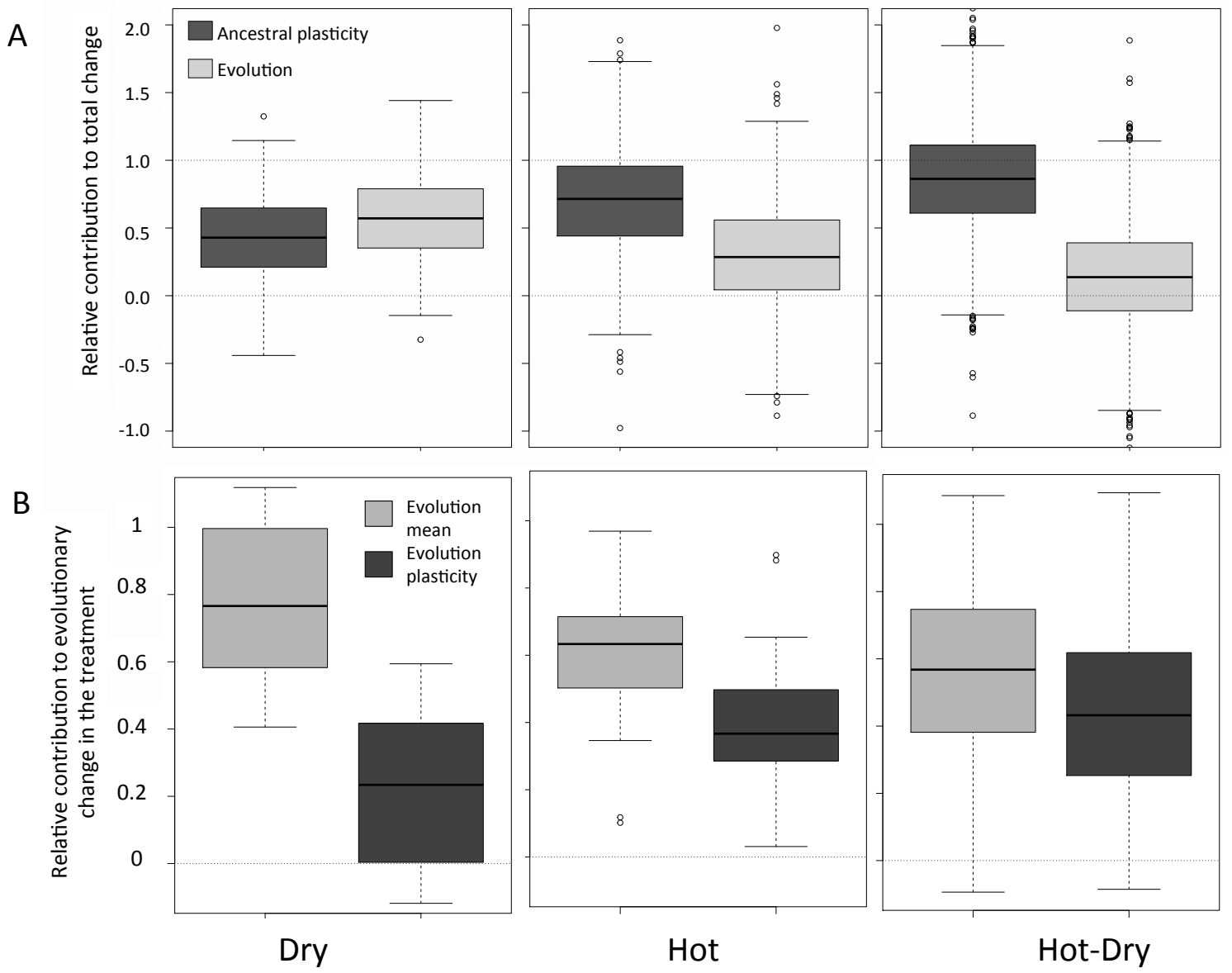

Figure 3: Relative contribution of ancestral plasticity and evolutionary change to total divergence for all genes showing significant total divergence in the DE analysis. Number of genes: Dry: 283, Hot: 1192, Hot-Dry: 2045 (A). Relative contribution of changes in the mean (shift in the intercept of reaction norm) and changes in plasticity (different slopes of reaction norms) to evolutionary differences between control-lines and selection lines in the treatment. Only genes with significant differences in the DE analysis were included. Number of genes: Dry: 18, Hot: 25, Hot-Dry: 55 (B). 


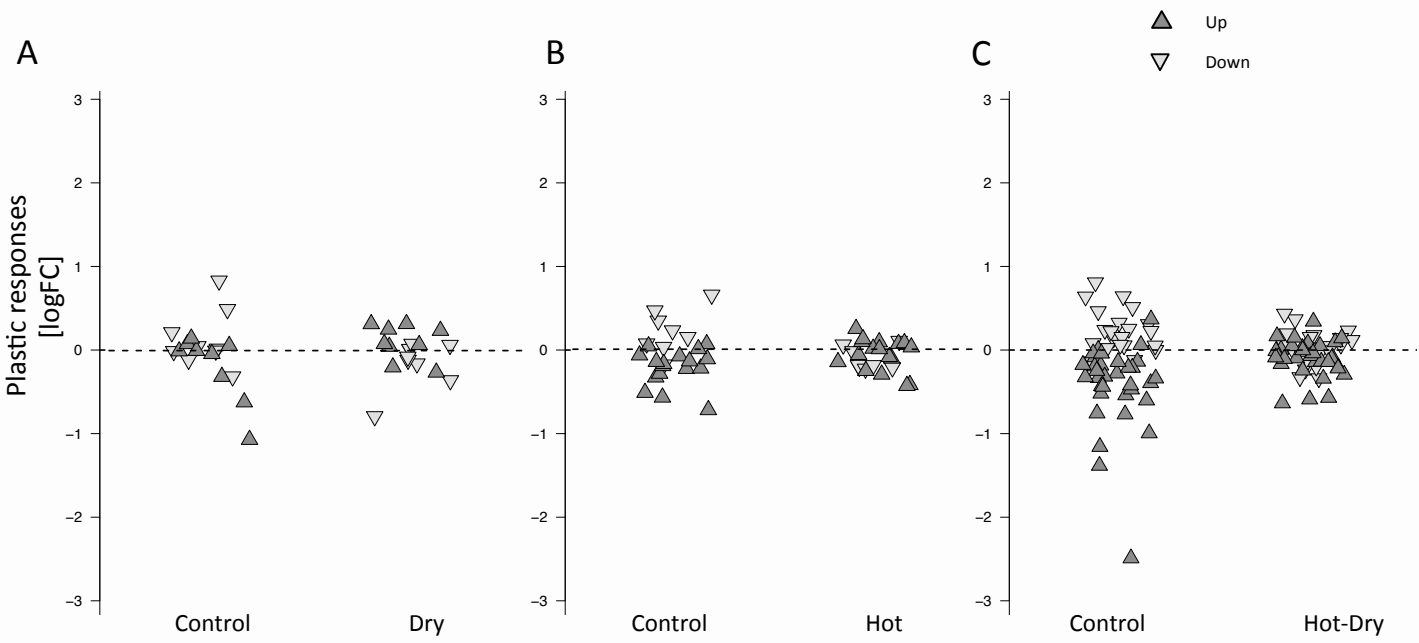

Selection

Figure 4: Plastic responses in control-lines and adapted selection-lines of genes showing significant evolutionary changes in expression in the treatments Dry (A), Hot (B) and Hot-Dry (C). Plastic responses in adapted selection lines are weaker (smaller logFC). Furthermore, plastic changes are mainly opposite to evolved changes. Genes that evolved to lower expression in the treatments (represented in lightgrey) show positive plastic changes in control-lines, i.e. they are up-regulated. Genes that evolved to higher expression levels (in darkgrey) are down-regulated in the non-adapted Control-Lines. 
A

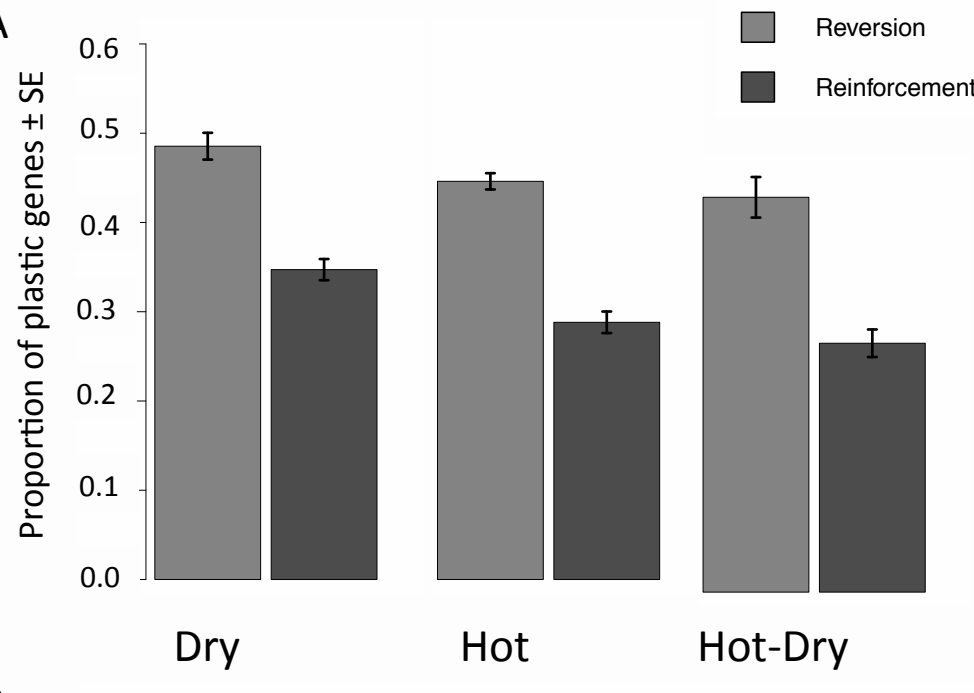

B

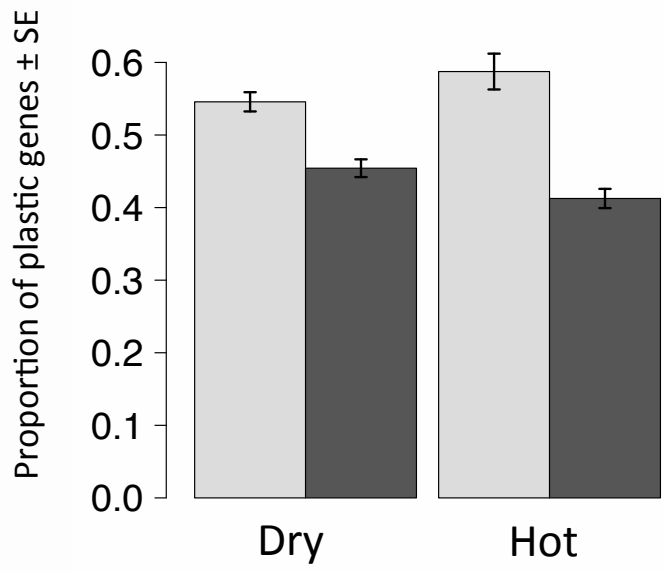

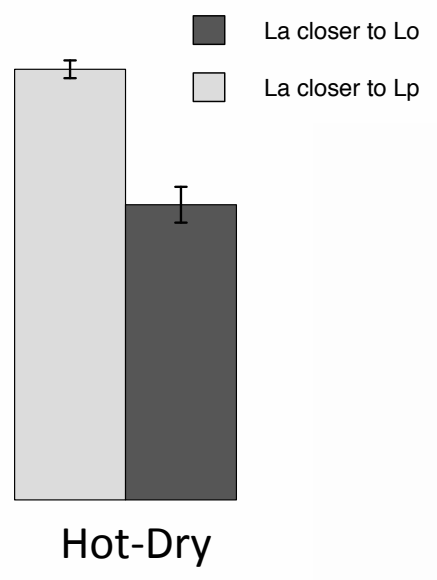

Figure 5: Comparison of plastic and evolutionary changes in gene expression in response to three environmental conditions. A: Proportion of genes (average over selection lines) showing a reversion or reinforcement of ancestral plasticity during evolution. B: Proportion of genes with expression levels after evolution (adapted stage La) closer to original levels (Lo, i.e. Control-lines in control conditions), or closer to plastic levels (Lp, i.e. expression levels of Control-lines in treatment). Only genes exhibiting substantial plastic changes ( $\mid$ Lp-Lo| $>20 \%$ of Lo) as well as evolutionary changes (La-Lp| > $20 \%$ of Lo ) were used for analysis. 
A

B

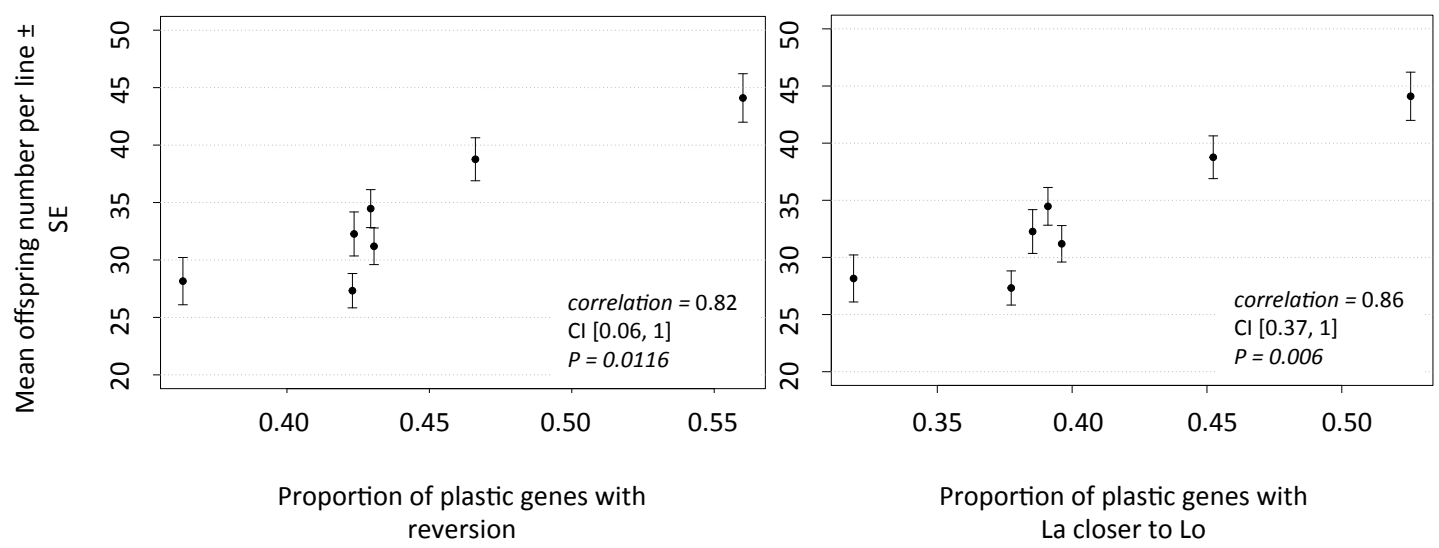
reversion La closer to Lo

Figure 6: Relationship between plastic and evolved changes in gene expression in response to hot-dry conditions. Expression levels of Control-lines in control conditions represent the ancestral stage Lo, Control-Lines in hot-dry conditions the plastic stage Lp and adapted Hot-Dry-lines in hot-dry conditions the adapted stage La. A: Relationship between the proportion of reversed plastic responses and mean fitness (=offspring number) per Hot-Dry line. B: Relationship between proportion of genes with La closer to Lo and mean fitness. Only genes exhibiting substantial plastic changes ( $\mid$ Lp-Lo $\mid>20 \%$ of Lo) as well as evolutionary changes (La-Lp $\mid>20 \%$ of Lo ) were used for analysis. Pvalues of the spearman correlations were obtained by 10,000 permutations. $95 \%$ Confidence intervals are based on a

Table 1: Number of sequenced replicate lines and individuals per selection and treatment, which were used for this study. Selection lines could adapt to conditions for 20 generations. Control (CT) conditions: $33^{\circ} \mathrm{C}, 70 \%$ relative humidity (r.h.); treatments: Dry (D): $33^{\circ} \mathrm{C}, 30 \%$ r.h.; Hot (H): $37^{\circ} \mathrm{C}, 70 \%$ r.h.; Hot-Dry (HD): $37^{\circ} \mathrm{C}, 30 \%$ r.h.

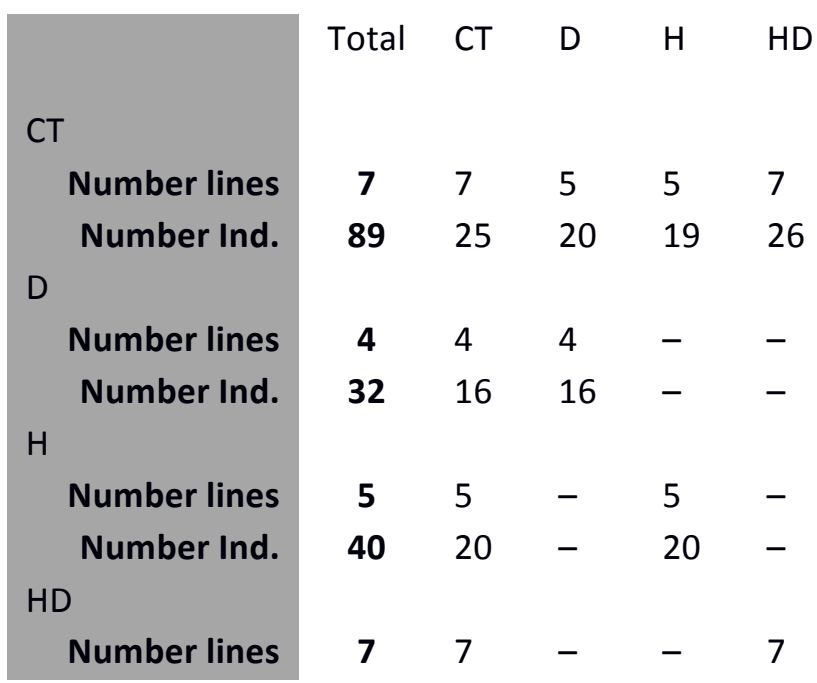


Table 2: Significantly differently expressed genes. For evolved differences, control-lines and selection-lines are compared within condition (e.g. Dry-lines vs Control-lines in Dry). Plastic response gives the number of genes that changed expression between control and treatment conditions (e.g. Dry-lines in Dry vs Dry-lines in control). Different plasticity gives the number of genes with significant different plastic responses in lines from different selection regimes. Analysis was conducted using the $\mathrm{R}$ package limma (Ritchie et al., 2015).

\begin{tabular}{|c|c|c|c|c|}
\hline & & Dry & Hot & Hot-Dry \\
\hline \multirow{3}{*}{$\begin{array}{l}\text { Evolved difference in } \\
\text { Control }\end{array}$} & down & 3 & 0 & 6 \\
\hline & up & 1 & 9 & 4 \\
\hline & total & 4 & 9 & 10 \\
\hline \multirow{3}{*}{$\begin{array}{l}\text { Evolved difference in } \\
\text { treatment }\end{array}$} & down & 9 & 8 & 22 \\
\hline & up & 9 & 17 & 33 \\
\hline & total & 18 & 25 & 55 \\
\hline \multirow{3}{*}{$\begin{array}{l}\text { Plastic response Control- } \\
\text { Lines }\end{array}$} & down & 209 & 1765 & 2451 \\
\hline & up & 156 & 1714 & 2200 \\
\hline & total & 365 & 3479 & 4651 \\
\hline \multirow{3}{*}{$\begin{array}{l}\text { Plastic response Selection- } \\
\text { Lines }\end{array}$} & down & 28 & 1417 & 1649 \\
\hline & up & 21 & 1381 & 1470 \\
\hline & total & 49 & 2798 & 3119 \\
\hline \multirow{3}{*}{$\begin{array}{l}\text { Total change (Selection-lines } \\
\text { in treatment vs Control-lines } \\
\text { in Control) }\end{array}$} & down & 154 & 564 & 1016 \\
\hline & up & 129 & 628 & 1029 \\
\hline & total & 283 & 1192 & 2045 \\
\hline different plasticity & total & 0 & 1 & 4 \\
\hline
\end{tabular}

Table 3: P-values obtained from permutation tests $(10,000$ permutations). Samples were randomly assigned to either control or treatment selection and differential expression analyses were repeated. Significance was assessed by calculating the proportion of permutations with more extreme values than the observed one. Control (CT) conditions: $33^{\circ} \mathrm{C}, 70 \%$ relative humidity r.h. Conditions in treatments: Dry (D): $33^{\circ} \mathrm{C}, 30 \%$ r.h.; Hot $(\mathrm{H}): 37^{\circ} \mathrm{C}, 70 \%$ r.h.; Hot-Dry (HD): $37^{\circ} \mathrm{C}, 30 \%$ r.h.

\section{Selection}

\begin{tabular}{l|ccc}
\hline & D & H & HD \\
\hline $\begin{array}{l}\text { CT-Lines have more genes with significant } \\
\text { plastic responses compared to adapted } \\
\text { selection lines }\end{array}$ & 0.2981 & 0.3037 & $\mathbf{0 . 0 3 2 5}$ \\
\hline $\begin{array}{l}\text { Magnitude of plastic response is higher in CT- } \\
\text { Lines }\end{array}$ & $\mathbf{0 . 0 4 9 3}$ & 0.1676 & $\mathbf{0 . 0 4 9 1}$ \\
\hline $\begin{array}{l}\text { Individuals from different selection regimes } \\
\text { show lower similarity in plastic responses }\end{array}$ & 0.8608 & 0.3914 & 0.5217 \\
\hline
\end{tabular}




\begin{tabular}{l|ccc}
\hline $\begin{array}{l}\text { Number of genes with significant differences in } \\
\text { expression levels is higher in treatment than in } \\
\text { CT conditions }\end{array}$ & 0.0095 & 0.0003 & 0.0031 \\
\hline $\begin{array}{l}\text { Differences in expression levels in CT } \\
\text { conditions and treatment are correlated }\end{array}$ & $<0.0001$ & $<0.0001$ & $<0.0001$ \\
\hline
\end{tabular}

\section{References}

Alvarez, M., Schrey, A. W., \& Richards, C. L. (2015). Ten years of transcriptomics in wild populations: What have we learned about their ecology and evolution? Molecular Ecology, 24(4), 710-725. doi:10.1111/mec.13055

Ayroles, J. F., Carbone, M. A., Stone, E. A., Jordan, K. W., Lyman, R. F., Magwire, M. M., ... Mackay, T. F. C. (2009). Systems genetics of complex traits in Drosophila melanogaster. Nature Genetics, 41(3), 299-307. doi:10.1038/ng.332

Barghi, N., Tobler, R., Nolte, V., Jakšić, A. M., Mallard, F., Otte, K. A., ... Schlötterer, C. (2019). Genetic redundancy fuels polygenic adaptation in Drosophila. PLoS Biology (Vol. 17). doi:10.1371/journal.pbio.3000128

Barton, M., Sunnucks, P., Norgate, M., Murray, N., \& Kearney, M. (2014). Co-gradient variation in growth rate and development time of a broadly distributed butterfly. PLOS ONE, 9(4), 1-8. doi:10.1371/journal.pone.0095258

Bates, D., Mächler, M., Bolker, B., \& Walker, S. (2015). Fitting Linear Mixed-Effects Models Using Ime4. Journal of Statistical Software, 67(1), 1-48. doi:10.18637/jss.v067.i01

Benjamini, Y., \& Hochberg, Y. (1995). Controlling the false discovery rate: a practical and powerful approach to multiple testing. Journal of the Royal Statistical Society. Series B (Methodological), 289-300.

Carroll, S. B. (2005). Evolution at Two Levels: On Genes and Form. PLoS Biology, 3(7), e245. doi:10.1371/journal.pbio.0030245

Chevin, L. M., Lande, R., \& Mace, G. M. (2010). Adaptation, plasticity, and extinction in a changing environment: Towards a predictive theory. PLoS Biology, 8(4). doi:10.1371/journal.pbio.1000357

Clemson, A. S., Sgrò, C. M., \& Telonis-Scott, M. (2016). Thermal plasticity in Drosophila melanogaster populations from eastern Australia: quantitative traits to transcripts. Journal of Evolutionary Biology, 29(12), 2447-2463. doi:10.1111/jeb.12969

Conover, D. O., Duffy, T. A., \& Hice, L. A. (2009). The covariance between genetic and environmental influences across ecological gradients: Reassessing the evolutionary significance of countergradient and cogradient variation. Annals of the New York Academy of Sciences, 1168(1), 100-129. doi:10.1111/j.1749-6632.2009.04575.x

Crispo, E. (2007). The Baldwin effect and genetic assimilation: Revisiting two mechanisms of evolutionary change mediated by phenotypic plasticity. Evolution, 61(11), 2469-2479. doi:10.1111/j.1558-5646.2007.00203.x

Dayan, D. I., Crawford, D. L., \& Oleksiak, M. F. (2015). Phenotypic plasticity in gene expression contributes to divergence of locally adapted populations of Fundulus heteroclitus. Molecular Ecology, 24(13), 3345-3359. doi:10.1111/mec.13188

de Jong, G. (2005). Evolution of phenotypic plasticity: patterns of plasticity and the emergence of ecotypes. New Phytologist, 166(2003), 101-118. doi:10.1111/j.1469-8137.2005.01322.x 
de Jong, Gerdien. (2005). Evolution of phenotypic plasticity: patterns of plasticity and the emergence of ecotypes. New Phytologist, 166(1), 101-118. doi:10.1111/j.14698137.2005.01322.x

Dobin, A., Davis, C. A., Schlesinger, F., Drenkow, J., Zaleski, C., Jha, S., ... Gingeras, T. R. (2013). STAR: ultrafast universal RNA-seq aligner. Bioinformatics, 29(1), 15-21.

Draghi, J. A., \& Whitlock, M. C. (2012). Phenotypic plasticity facilitates mutational variance, genetic variance, and evolvability along the major axis of environmental variation. Evolution, 66(9), 2891-2902. doi:10.1111/j.1558-5646.2012.01649.x

Fangue, N. A., Hofmeister, M., \& Schulte, P. M. (2006). Intraspecific variation in thermal tolerance and heat shock protein gene expression in common killifish, Fundulus heteroclitus. The Journal of Experimental Biology, 209(Pt 15), 2859-72. doi:10.1242/jeb.02260

Feder, M. E., \& Hoffman, G. E. (1999). Heat-shock proteins, molecular chaperones, and the stress response: evolutionary and ecological physiology. Annual Review of Physiology, 61, 243282.

Feder, M. E., \& Krebs, R. A. (1998). Natural and Genetic Engineering of the Heat-Shock Protein Hsp70 in Drosophila melanogaster : Consequences for Thermotolerance. American Zoologist, 38(3), 503-517. doi:10.1093/icb/38.3.503

Fierst, J. L. (2011). A history of phenotypic plasticity accelerates adaptation to a new environment. Journal of Evolutionary Biology, 24(9), 1992-2001. doi:10.1111/j.14209101.2011.02333.x

Fitzpatrick, B. M. (2012). Underappreciated consequences of phenotypic plasticity for ecological speciation. International Journal of Ecology, 2012, 32-37. doi:10.1155/2012/256017

Forsman, A. (2015). Rethinking phenotypic plasticity and its consequences for individuals, populations and species. Heredity, 115(4), 276-284. doi:10.1038/hdy.2014.92

Garland, T., \& Kelly, S. A. (2008). Phenotypic plasticity and experimental evolution. Journal of Experimental Biology, 211(16), 2725-2725. doi:10.1242/jeb.022673

Ghalambor, C. K., Hoke, K. L., Ruell, E. W., Fischer, E. K., Reznick, D. N., \& Hughes, K. A. (2015). Non-adaptive plasticity potentiates rapid adaptive evolution of gene expression in nature. Nature, 525(7569), 372-375. doi:10.1038/nature15256

Ghalambor, C. K., McKay, J. K., Carroll, S. P., \& Reznick, D. N. (2007). Adaptive versus nonadaptive phenotypic plasticity and the potential for contemporary adaptation in new environments. Functional Ecology, 21(3), 394-407. doi:10.1111/j.1365-2435.2007.01283.x

Gibbons, T. C., Metzger, D. C. H., Healy, T. M., \& Schulte, P. M. (2017). Gene expression plasticity in response to salinity acclimation in threespine stickleback ecotypes from different salinity habitats. Molecular Ecology, 26(10), 2711-2725. doi:10.1111/mec.14065

Gibson, G. (2008). The environmental contribution to gene expression profiles. Nature Reviews Genetics, 9(8), 575.

Gibson, G., \& Dworkin, I. (2004). Uncovering cryptic genetic variation. Nature Reviews Genetics, 5(9), 681-690. doi:10.1038/nrg1426

Grether, G. F. (2005). Environmental Change, Phenotypic Plasticity, and Genetic Compensation. The American Naturalist, 166(4), E115-E123. doi:10.1086/432023

Guo, J., Liu, R., Huang, L., Zheng, X. M., Liu, P. L., Du, Y. S., ... Ge, S. (2016). Widespread and adaptive alterations in genome-wide gene expression associated with ecological divergence of two oryza species. Molecular Biology and Evolution, 33(1), 62-78. doi:10.1093/molbev/msv196

Hasan, M. M., DeFaveri, J., Kuure, S., Dash, S. N., Lehtonen, S., Merilä, J., \& McCairns, R. J. S. (2017). Sticklebacks adapted to divergent osmotic environments show differences in plasticity for kidney morphology and candidate gene expression. The Journal of 
Hatakeyama, M., Opitz, L., Russo, G., Qi, W., Schlapbach, R., \& Rehrauer, H. (2016). SUSHI: An exquisite recipe for fully documented, reproducible and reusable NGS data analysis. BMC Bioinformatics, 17(1), 1-9. doi:10.1186/s12859-016-1104-8

Ho, W.-C., \& Zhang, J. (2018). Evolutionary adaptations to new environments generally reverse plastic phenotypic changes. Nature Communications, 9(1), 1-11. doi:10.1038/s41467-01702724-5

Ho, W.-C., \& Zhang, J. (2019). Genetic gene expression changes during environmental adaptations tend to reverse plastic changes even after the correction for statistical nonindependence. Molecular Biology and Evolution. doi:10.1093/molbev/msz002

Hodgins-Davis, A., \& Townsend, J. P. (2009). Evolving gene expression: from $G$ to $E$ to $G \times E$. Trends in Ecology and Evolution, 24(12), 649-658. doi:10.1016/j.tree.2009.06.011

Huang, Y., \& Agrawal, A. F. (2016). Experimental Evolution of Gene Expression and Plasticity in Alternative Selective Regimes. PLoS Genetics, 12(9), 1-23. doi:10.1371/journal.pgen.1006336

Krebs, R. A., \& Feder, M. E. (1997). Natural Variation in the Expression of the Heat-Shock Protein HSP70 in a Population of Drosophila melanogaster and Its Correlation with Tolerance of Ecologically Relevant Thermal Stress. Evolution, 51(1), 173. doi:10.2307/2410970

Kregel, K. C. (2002). Invited Review: Heat shock proteins: modifying factors in physiological stress responses and acquired thermotolerance. Journal of Applied Physiology, 92(5), 2177-2186. doi:10.1152/japplphysiol.01267.2001

Kültz, D. (2005). Molecular and Evolutionary Basis of the Cellular Stress Response. Annual Review of Physiology, 67(1), 225-257. doi:10.1146/annurev.physiol.67.040403.103635

Kuznetsova, A., Brockhoff, P. B., \& Christensen, R. H. B. (2017). ImerTest Package: Tests in Linear Mixed Effects Models. Journal of Statistical Software, 82(13), 1-26. doi:10.18637/jss.v082.i13

Lande, R. (2009). Adaptation to an extraordinary environment by evolution of phenotypic plasticity and genetic assimilation. Journal of Evolutionary Biology, 22(7), 1435-1446. doi:10.1111/j.1420-9101.2009.01754.x

Laugen, A. T., Laurila, A., Räsänen, K., \& Merilä, J. (2003). Latitudinal countergradient variation in the common frog ( Rana temporaria ) development rates - evidence for local adaptation. $J$. Evol., 16(5), 996-1005.

Law, C. W., Chen, Y., Shi, W., \& Smyth, G. K. (2014). voom: precision weights unlock linear model analysis tools for RNA-seq read counts. Genome Biology, 15(2), R29. doi:10.1186/gb-201415-2-r29

Lenth, R. V. (2016). Least-Squares Means: The R Package Ismeans. Journal of Statistical Software, 69(1), 1-33. doi:10.18637/jss.v069.i01

Levine, M. T., Eckert, M. L., \& Begun, D. J. (2011). Whole-genome expression plasticity across tropical and temperate Drosophila melanogaster populations from eastern Australia. Molecular Biology and Evolution, 28(1), 249-256. doi:10.1093/molbev/msq197

Levis, N. A., \& Pfennig, D. W. (2016). Evaluating 'Plasticity-First' Evolution in Nature : Key Criteria and Empirical Approaches. Trends in Ecology \& Evolution, 31(7), 563-574. doi:10.1016/j.tree.2016.03.012

Li, A., Li, L., Song, K., Wang, W., \& Zhang, G. (2017). Temperature, energy metabolism, and adaptive divergence in two oyster subspecies. Ecology and Evolution, 7(16), 6151-6162. doi:10.1002/ece3.3085

Liao, Y., Smyth, G. K., \& Shi, W. (2014). FeatureCounts: An efficient general purpose program for assigning sequence reads to genomic features. Bioinformatics, 30(7), 923-930. doi:10.1093/bioinformatics/btt656 

component in Drosophila depend on environmental temperature. American Naturalist, 169(2), 175-183. doi:10.1086/510632

841

Lohman, B. K., Stutz, W. E., \& Bolnick, D. I. (2017). Gene expression stasis and plasticity following migration into a foreign environment. Molecular Ecology, 26(18), 4657-4670. doi:10.1111/mec.14234

Mahroof, R., Zhu, K. Y., \& Subramanyam, B. (2005). Changes in Expression of Heat Shock Proteins in Tribolium castaneum (Coleoptera: Tenebrionidae) in Relation to Developmental Stage, Exposure Time, and Temperature. Annals of the Entomological Society of America, 98(1), 100-107. doi:10.1603/0013-8746(2005)098[0100:CIEOHS]2.0.CO;2

Mäkinen, H., Papakostas, S., Vøllestad, L. A., Leder, E. H., \& Primmer, C. R. (2016). Plastic and evolutionary gene expression responses are correlated in European grayling (Thymallus thymallus) subpopulations adapted to different thermal environments. In Journal of Heredity (Vol. 107, pp. 82-89). doi:10.1093/jhered/esv069

Mallard, F., Jakšić, A. M., \& Schlötterer, C. (2018). Contesting the evidence for non-adaptive plasticity. Nature, 555(7698), E21.

McCairns, R. J. S. S., \& Bernatchez, L. (2009). Adaptive divergence between freshwater and marine sticklebacks: insights into the role of phenotypic plasticity from an integrated analysis of candidate gene expression. Evolution, 64(4), 1029-1047. doi:10.1111/j.15585646.2009.00886.x

McGraw, E. A., Ye, Y. H., Foley, B., Chenoweth, S. F., Higgie, M., Hine, E., \& Blows, M. W. (2011). High-dimensional variance partitioning reveals the modular genetic basis of adaptive divergence in gene expression during reproductive character displacement. Evolution, 65(11), 3126-3137. doi:10.1111/j.1558-5646.2011.01371.x

Milutinović, B., Stolpe, C., Peuß, R., Armitage, S. A. O., \& Kurtz, J. (2013). The Red Flour Beetle as a Model for Bacterial Oral Infections. PLOS ONE, 8(5). doi:10.1371/journal.pone.0064638

Molina-Montenegro, M. A., Peñuelas, J., Munné-Bosch, S., \& Sardans, J. (2012). Higher plasticity in ecophysiological traits enhances the performance and invasion success of Taraxacum officinale (dandelion) in alpine environments. Biological Invasions, 14(1), 21-33. doi:10.1007/s10530-011-0055-2

Morris, M. R. J., Richard, R., Leder, E. H., Barrett, R. D. H., Aubin-Horth, N., \& Rogers, S. M. (2014). Gene expression plasticity evolves in response to colonization of freshwater lakes in threespine stickleback. Molecular Ecology, 23(13), 3226-3240. doi:10.1111/mec.12820

Narum, S. R., \& Campbell, N. R. (2015). Transcriptomic response to heat stress among ecologically divergent populations of redband trout. BMC Genomics, 16(1), 103. doi:10.1186/s12864-015-1246-5

Nussey, D. H., Postma, E., Gienapp, P., \& Visser, M. E. (2005). Selection on heritable phenotypic plasticity in a wild bird population. Science, 310(5746), 304-306. doi:10.1126/science.1117004

Passow, C. N., Henpita, C., Shaw, J. H., Quackenbush, C. R., Warren, W. C., Schartl, M., ... Tobler, $M$. (2017). The roles of plasticity and evolutionary change in shaping gene expression variation in natural populations of extremophile fish. Molecular Ecology, 26(22), 63846399. doi:10.1111/mec.14360

Pavey, S. A., Collin, H., Nosil, P., \& Rogers, S. M. (2010). The role of gene expression in ecological speciation. Annals of the New York Academy of Sciences, 1206, 110-129. doi:10.1111/j.1749-6632.2010.05765.x

Pfennig, D. W., Wund, M. A., Snell-Rood, E. C., Cruickshank, T., Schlichting, C. D., \& Moczek, A. P. (2010). Phenotypic plasticity's impacts on diversification and speciation. Trends in Ecology and Evolution, 25(8), 459-467. doi:10.1016/j.tree.2010.05.006 
Pichancourt, J. B., \& van Klinken, R. D. (2012). Phenotypic plasticity influences the size, shape and dynamics of the geographic distribution of an invasive plant. PLOS ONE, 7(2), e32323. doi:10.1371/journal.pone.0032323

Pigliucci, M., Murren, C. J., \& Schlichting, C. D. (2006). Phenotypic plasticity and evolution by genetic assimilation. Journal of Experimental Biology, 209(12), 2362-2367. doi:10.1242/jeb.02070

Pigliucci, Massimo. (2005). Evolution of phenotypic plasticity: Where are we going now? Trends in Ecology and Evolution, 20(9), 481-486. doi:10.1016/j.tree.2005.06.001

Price, T. D., Qvarnstrom, A., \& Irwin, D. E. (2003). The role of phenotypic plasticity in driving genetic evolution. Proceedings of the Royal Society B: Biological Sciences, 270(1523), 14331440. doi:10.1098/rspb.2003.2372

Price, Trevor D., Qvarnström, A., \& Irwin, D. E. (2003). The role of phenotypic plasticity in driving genetic evolution. Proceedings of the Royal Society B: Biological Sciences, 270(1523), 14331440. doi:10.1098/rspb.2003.2372

R Core Team. (2017). R Core Team (2017). R: A language and environment for statistical computing. $R$ Foundation for Statistical Computing, Vienna, Austria. URL Http://Www.RProject.Org/.

Ragland, G. J., Almskaar, K., Vertacnik, K. L., Gough, H. M., Feder, J. L., Hahn, D. A., \& Schwarz, D. (2015). Differences in performance and transcriptome-wide gene expression associated with Rhagoletis (Diptera: Tephritidae) larvae feeding in alternate host fruit environments. Molecular Ecology, 24(11), 2759-2776. doi:10.1111/mec.13191

Ritchie, M. E., Phipson, B., Wu, D., Hu, Y., Law, C. W., Shi, W., \& Smyth, G. K. (2015). limma powers differential expression analyses for RNA-sequencing and microarray studies. Nucleic Acids Research, 43(7), e47-e47. doi:10.1093/nar/gkv007

Robinson, M. D., McCarthy, D. J., \& Smyth, G. K. (2010). edgeR: a Bioconductor package for differential expression analysis of digital gene expression data. Bioinformatics, 26(1), 139140. doi:10.1093/bioinformatics/btp616

Romero, I. G., Ruvinsky, I., \& Gilad, Y. (2012). Comparative studies of gene expression and the evolution of gene regulation. Nature Reviews Genetics, 13(7), 505-516. doi:10.1038/nrg3229

Schaum, E., Rost, B., Millar, A. J., \& Collins, S. (2013). Variation in plastic responses of a globally distributed picoplankton species to ocean acidification. Nature Climate Change, 3(3), 298302. doi:10.1038/nclimate 1774

Scheiner, S. M. (1993). Genetics and Evolution of Phenotypic Plasticity. Annual Review of Ecology and Systematics, 24(1), 35-68. doi:10.1146/annurev.es.24.110193.000343

Schwenke, R. A., Lazzaro, B. P., \& Wolfner, M. F. (2016). Reproduction-Immunity Trade-Offs in Insects. Annual Review of Entomology, 61(1), 239-256. doi:10.1146/annurev-ento-010715023924

Shaw, J. R., Hampton, T. H., King, B. L., Whitehead, A., Galvez, F., Gross, R. H., ... Stanton, B. A. (2014). Natural Selection Canalizes Expression Variation of Environmentally Induced Plasticity-Enabling Genes. Molecular Biology and Evolution, 31(11), 3002-3015. doi:10.1093/molbev/msu241

Sikkink, K. L., Reynolds, R. M., Ituarte, C. M., Cresko, W. A., \& Phillips, P. C. (2014). Rapid Evolution of Phenotypic Plasticity and Shifting Thresholds of Genetic Assimilation in the Nematode Caenorhabditis remanei. G3: Genes/Genomes/Genetics, 4(6), 1103-1112. doi:10.1534/g3.114.010553

Silbermann, R., \& Tatar, M. (2000). Reproductive costs of heat shock protein in transgenic Drosophila melanogaster. Evolution, 54(6), 2038-2045. doi:10.1111/j.00143820.2000.tb01247.x 
Smyth, G. K., Michaud, J., \& Scott, H. S. (2005). Use of within-array replicate spots for assessing differential expression in microarray experiments. Bioinformatics, 21(9), 2067-2075. doi:10.1093/bioinformatics/bti270

Sokoloff, A. (1972). The biology of Tribolium with special emphasis on genetic aspects I. Clarendon Press and Oxford Univ. Press, Oxford. doi:10.1086/407731

Sokolova, I. M. (2013). Energy-limited tolerance to stress as a conceptual framework to integrate the effects of multiple stressors. Integrative and Comparative Biology, 53(4), 597-608. doi:10.1093/icb/ict028

Sørensen, J G, Dahlgaard, J., \& Loeschcke, V. (2001). Genetic variation in thermal tolerance among natural populations of Drosophila buzzatii: down regulation of Hsp70 expression and variation in heat stress resistance traits. Functional Ecology, 15(3), 289-296. doi:10.1046/j.1365-2435.2001.00525.x

Sørensen, Jesper Givskov, Kristensen, T. N., \& Loeschcke, V. (2003). The evolutionary and ecological role of heat shock proteins. Ecology Letters, 6(11), 1025-1037. doi:10.1046/j.1461-0248.2003.00528.x

Stearns, S. C., \& Kawecki, T. J. (2006). Fitness Sensitivity and the Canalization of Life-History Traits. Evolution, 48(5), 1438. doi:10.2307/2410238

Stoks, R., Govaert, L., Pauwels, K., Jansen, B., \& De Meester, L. (2016). Resurrecting complexity: The interplay of plasticity and rapid evolution in the multiple trait response to strong changes in predation pressure in the water flea Daphnia magna. Ecology Letters, 19(2), 180-190. doi:10.1111/ele.12551

Townsend, J. P., Cavalieri, D., \& Hartl, D. L. (2003). Population genetic variation in genome-wide gene expression. Molecular Biology and Evolution, 20(6), 955-963. doi:10.1093/molbev/msg106

Via, S. (1993). Adaptive Phenotypic Plasticity: Target or By-Product of Selection in a Variable Environment? The American Naturalist, 142(2), 352-365. doi:10.1086/285542

von Heckel, K., Stephan, W., \& Hutter, S. (2016). Canalization of gene expression is a major signature of regulatory cold adaptation in temperate Drosophila melanogaster. $B M C$ Genomics, 17(1), 1-14. doi:10.1186/s12864-016-2866-0

Whitehead, A., \& Crawford, D. L. (2006). Neutral and adaptive variation in gene expression. Proceedings of the National Academy of Sciences of the United States of America|r, 103(14), 5425-5430. doi:10.1073/pnas.0507648103

Wray, G. A. (2007). The evolutionary significance of cis-regulatory mutations. Nature Reviews Genetics, 8(3), 206-216. doi:10.1038/nrg2063

Wund, M. A. (2012). Assessing the impacts of phenotypic plasticity on evolution. Integrative and Comparative Biology, 52(1), 5-15. doi:10.1093/icb/ics050

Yampolsky, L. Y., Glazko, G. V., \& Fry, J. D. (2012). Evolution of gene expression and expression plasticity in long-term experimental populations of Drosophila melanogaster maintained under constant and variable ethanol stress. Molecular Ecology, 21(17), 4287-4299. doi:10.1111/j.1365-294X.2012.05697.x

Yampolsky, L. Y., Zeng, E., Lopez, J., Williams, P. J., Dick, K. B., Colbourne, J. K., \& Pfrender, M. E. (2014). Functional genomics of acclimation and adaptation in response to thermal stress in Daphnia. BMC Genomics, 15(1), 1-12. doi:10.1186/1471-2164-15-859

Yeh, P. J., \& Price, T. D. (2004). Adaptive Phenotypic Plasticity and the Successful Colonization of a Novel Environment. The American Naturalist, 164(4), 531-542. doi:10.1086/423825

Zhao, L., \& Jones, W. A. (2012). Expression of heat shock protein genes in insect stress responses. Invertebrate Survival Journal, 9(1), 93-101. 